Mon. Not. R. Astron. Soc. 000, 120 (2015) Printed 26 July $2018 \quad$ (MN LATEX style file v2.2)

\title{
The Shape of Dark Matter Haloes I. HI Observations of Edge-on Galaxies
}

\author{
S. P. C. Peters ${ }^{1}$, P. C. van der Kruit ${ }^{1 \star}$, R. J. Allen ${ }^{2}$ and K. C. Freeman ${ }^{3}$ \\ ${ }^{1}$ Kapteyn Astronomical Institute, University of Groningen, P.O.Box 800, 9700AV Groningen, the Netherlands \\ ${ }^{2}$ Space Telescope Science Institute, 3700 San Martin Drive, Baltimore, MD 21218, USA \\ ${ }^{3}$ Research School of Astronomy and Astrophysics The Australian National University, Cotter Road Weston Creek, ACT 2611, \\ Australia
}

Accepted 2015 month xx. Received 2015 Month xx; in original form 2015 Month xx

\begin{abstract}
We present neutral hydrogen observations for a sample of eight nearby, late-type, edgeon galaxies. All of the galaxies have been well resolved in the radial direction, while six have also been well resolved in the vertical direction. We find that each of the galaxies has approximately the same maximum surface brightness temperature throughout its disc. We argue that self-absorption is the main cause of this phenomenon and that subsequent decompositions will require a treatment of this.
\end{abstract}

Key words: galaxies: haloes, galaxies: kinematics and dynamics, galaxies: photometry, galaxies: spiral, galaxies: structure

\section{INTRODUCTION TO THE SERIES}

Rotation curve of galaxies remain flat at large radii, implying that the amount of mass-to-light increases drastically with radius from the centre. This first indication, in the early seventies by Freeman (1970), was that in two galaxies the observed HI -rotation curves did not decline as the exponential disk of starlight would predict. This was quickly followed by observed flat rotation curves beyond the optical disks by workers as Mort Roberts, Albert Bosma and others at $21 \mathrm{~cm}$, and by Vera Rubin and others in radial velocities of HII-regions within the optical boundaries. The presence of a halo of dark matter that was assumed to be more or less spherical. Not much later it was realised that for galaxies to remain stable, an additional dark matter component was required. Analysis of the thickness of the HI layer in NGC 891 (van der Kruit 1981), showed that this dark matter could not reside in the disc and would form a more or less spherical structure in which the visible galaxy would be embedded. These developments have been discussed by many authors, e.g. O'Brien et al. (2010), van der Kruit \& Freeman (2011) and Bland-Hawthorn (2014).

To this date however, the nature of the dark matter remains elusive. By understanding the dark matter halo in detail, it will be possible for physicists to place stronger constraints on the theoretical nature of the dark matter. In this series of papers, which is a major part of the $\mathrm{PhD}$ thesis of the first author, Peters (2014), we continue the work by

\footnotetext{
* For more information, please contact P.C. van der Kruit at vdkruit@astro.rug.nl.
}

O'Brien et al. (2010) and O'Brien et al. (2010a b c), who set out to measure the shape of the dark matter halo around edge-on galaxies using the flaring of the HI layer.

Because of their side-on orientation, edge-on galaxies offer a unique perspective on the structure of galaxies. Due to the random orientation of galaxies in space, only a fraction is seen edge-on. Our Galaxy can be considered a prime example of an edge-on galaxy. There are many advantages to the edge-on perspective. For example, the longer lines-of-sight allow the study of the baryonic content at very low volume densities. This led van der Kruit (1979) to the discovery that most stellar discs have a truncation in their outer regions, beyond which the stellar density rapidly drops to zero. In face-on galaxies only modern techniques have been able to establish the existence of truncation, see Peters et al. (2015) and Peters (2014).

Another advantage of the edge-on perspective is that they allow for the study of the structure of the discs as function of height above the plane. This advantage led to the discovery of thick stellar discs, using surface photometry. Evidence for this additional mass component has been detected first in S0 galaxies (Burstein 1979, Tsikoudi 1980). in the Galaxy, and then Gilmore \& Reid (1983) using stellar counts. In face-on galaxies, the vertical direction is integrated along the line-of-sight, making the thick and thin disc hard to disentangle.

While offering a unique perspective on the vertical structure of the disc, each line-of-sight also forms a superposition of light emitted at various radii, as the line crosses through the disc. Decomposition of the stellar discs is required before the galaxy can be understood as function of 
radius $R$ and height $z$. Many authors have thus developed new and ever more sophisticated methods to disentangle this information (e.g. van der Kruit \& Searle 1981, Pohlen et al. 2007, Comerón et al. 2011). The most modern methods are able to model the full spectrum from near infrared to optical and can account for the dust reprocessing of the light de Geyter et al. 2013).

For neutral hydrogen, the situations is even more complex. Where most optical and infrared emission comes in the form of very broad lines and blackbody emission, the hydrogen line is very narrow. Because of this, the most prominent source of broadening of the HI line emission of a galaxy is due to the Doppler shift from the bulk motion of the gas, in particular due to the rotation around the galaxy and the turbulence of the gas. The broadening due to the turbulence of the gas (also known as the velocity dispersion) is typically of order $10 \mathrm{~km} / \mathrm{s}$ and is low compared to the rotational velocity of the galaxy, which is typically of order $100 \mathrm{~km} / \mathrm{s}$ or more. If the observation of an edge-on galaxy is well resolved spatially and in velocity, for example using a radio interferometer, the line-of-sight velocity $v_{\text {los }}$ offers an additional constraint on a decomposition.

We begin in this first paper of the series with an analysis of the neutral hydrogen (HI ) content of eight edge-on galaxies. One of the main questions that arises in this paper is the possible impact that HI self-absorption of the gas can have on any subsequently derived property. Traditionally this topic has largely been ignored, but we argue that it has to be addressed before any sensible statement can be made about the dark matter halo from HI data. The currently available modelling tools are unable to treat for $\mathrm{HI}$ self-absorption, which is why we introduce a new software tool called GALACTUS in paper II. This tool allows the users to perform a (Bayesian) fit to the HI data cubes. We use this tool to model the face-on galaxies NGC 2403. Projecting the results to an edge-on model, we find that the maximum temperature maps from this paper should indeed have been higher, concluding that self-absorption indeed is important. We also demonstrate how the missing mass fraction due to the HI self-absorption increases with inclination.

In paper III, we return to the radio cubes presented here. Using a series of simulated HI cubes, we demonstrate that with our new software it is possible to analyse the edgeon galaxies. We also demonstrate how treating the $\mathrm{HI}$ as optically thin can lead to errors in the measured properties. The rest of the paper is dedicated to analysing the eight edge-on galaxies.

Paper IV is dedicated to modelling the stellar and dust contents of these edge-on galaxies. We use the software package FITSKIRT to model the galaxies in multiple wavelengths using optical and near-infrared observations.

In paper $\mathrm{V}$, we use these $\mathrm{HI}$ and stellar results to model the theoretical hydrostatics of the edge-on galaxies. Comparing the theoretical and observed hydrostatics enables us to measure the gravitational potential of the dark matter halo in both the radial and vertical direction. We fit dark matter haloes to the best five out of our initial eight galaxies. Three galaxies have dark matter haloes that are nearly spherical, while two more have strongly prolate haloes. We did not find any strongly oblate haloes.

\section{INTRODUCTION TO THIS PAPER}

The first spatially resolved HI observations of edge-on galaxies came in the late seventies, but lacked sufficient quality for a detailed analysis (Sancisi 1976, Weliachew et al. 1978). That became possible with the subsequent observation of NGC 891 by Sancisi \& Allen (1979), a nearby edge-on galaxy similar to the Galaxy (van der Kruit 1984). Sancisi \& Allen modelled both the rotation curve and the thickness of the disc, concluding that the thickness was less than one kpc in the inner parts, but flaring out to $1-2 \mathrm{kpc}$ thickness at $20-24$ kpc radius. It was shown by van der Kruit (1981) that the thickness of this HI layer should and does increase exponentially with radius, which was later confirmed by Rupen (1991). Since then, NGC 891 has remained a prime target for subsequent observations, each time improving the quality of the data and revealing new structure, such as the presence of an HI well below and above the disc (Swaters et al. 1997, Oosterloo et al. 2007). We also note that this gas in NGC 891 is lagging behind the HI in the disk. We refer also to the work of Matthews \& Wood (2003) on one of the galaxies in our sample, UGC 7321. These important developments produce effects that will have to be kept in mind.

Olling (1996) was the first to derive the radial HI surface density of an edge-on, by decomposing the galaxy using inverse Abel transformation in a technique inspired by the work on face-on galaxies by Warmels (1988). While the velocity dispersion in face-on galaxies can be measured more or less directly, the superposition of gas in edge-on systems at various radii requires a careful decomposition of the galaxy. In the same paper, Olling (1996) also used the outer envelope of the $\mathrm{XV}$-diagram to measure the velocity dispersion variation in NGC 4244, which was found to be consistent with a velocity dispersion of roughly $8.5 \mathrm{~km} / \mathrm{s}$. O'Brien et al. (2010b) modelled the full XV-diagram for eight edgeon galaxies and found that most systems display HI velocity dispersions of 6.5 to $7.5 \mathrm{~km} / \mathrm{s}$ and all except one show radial structure in this property.

We ultimately aim to measure the shape of the dark matter halo around edge-on galaxies. By carefully modelling the theoretical hydrostatics of the neutral hydrogen and stellar components in each galaxy, and comparing this with the observed hydrostatics, it is possible to discern the effects due to the dark matter halo. This is a continuation of the project started in O'Brien et al. (2010 a b c), who modelled these components for galaxy UGC7321 and found a near spherical halo. In this paper, we present the HI observations for eight edge-on galaxies, similar to the sample of O'Brien et al. (2010). A key question from O'Brien et al. (2010) concerns the possible effects of self-absorption of the neutral hydrogen. We begin this paper by re-addressing this issue.

\section{A TOY MODEL}

A continuing concern throughout this series of papers will be the role of the HI self-absorption (sometimes referred to as HISA in literature) on the observations. In our own Galaxy, many lines of sight are known to become optically thick within several hundred parsec, with faint HISA features visible in all direction where the background emission 

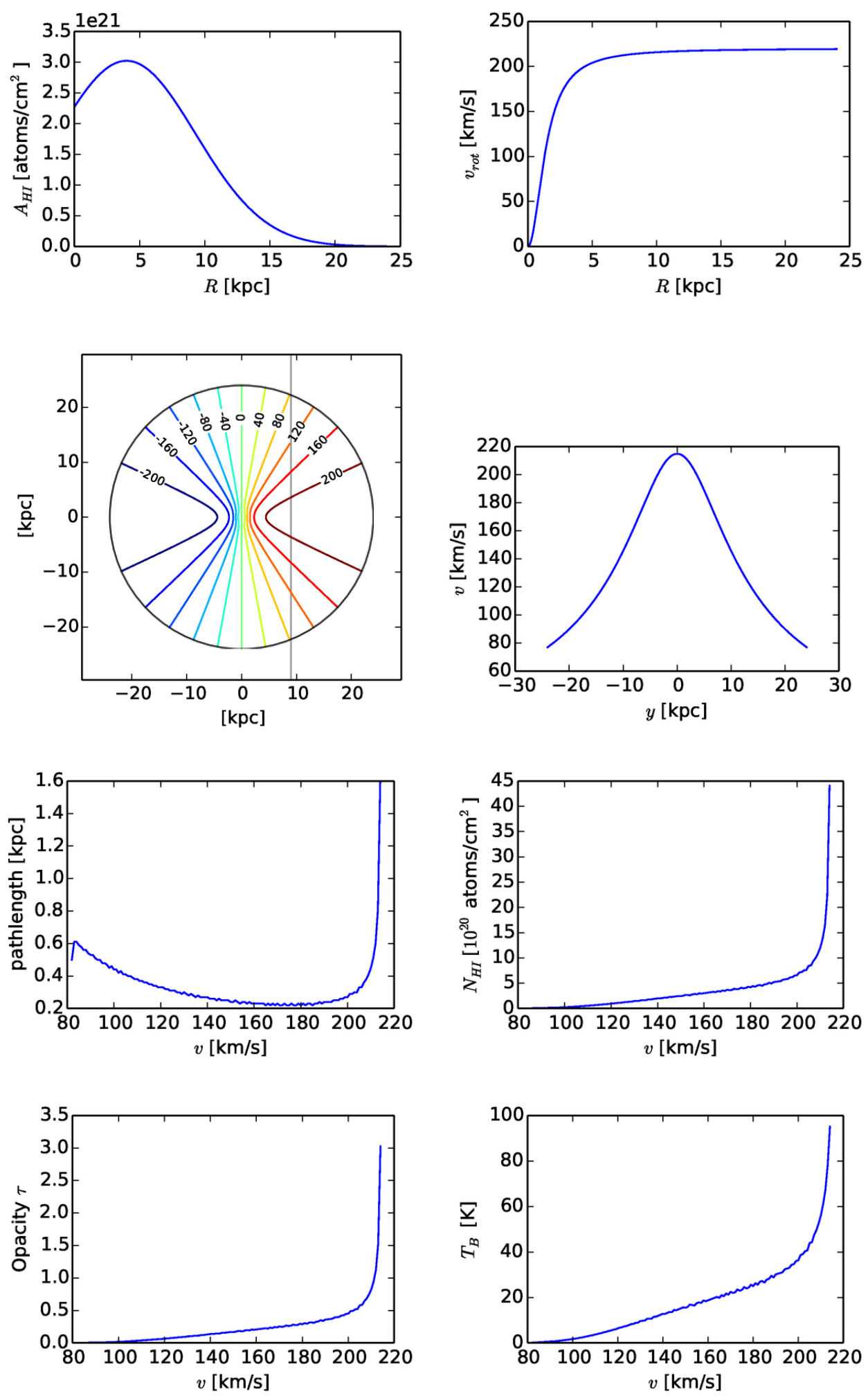

Figure 1. Tracing a ray through a toy model. Top row: Face-on surface density and rotation curve of the toy model. Second row left: Spider diagram of the projected velocity at each position in the disc, with the grey vertical line demonstrating the ray at nine kpc. Second row right: projected velocity along the ray. Third row left: The distance crossed by the ray in each projected velocity. Third row right: The amount of $\mathrm{HI}$ atoms at each projected velocity. Bottom row left: Absorption coefficient $\tau_{\nu}$ with velocity. Bottom row right: Observed brightness with velocity. 


\begin{tabular}{lll} 
Parameter & Function & Value \\
\hline \hline Inclination & & $i=90^{\circ}$ \\
Thickness of disc & & FWHM $=700 \mathrm{kpc}$ \\
Systemic velocity & $v_{\text {Sys }}=0 \mathrm{~km} / \mathrm{s}$ \\
Rotation curve & $v_{\infty}=220 \mathrm{~km} / \mathrm{s}$ \\
& & $a=1.4 \mathrm{kpc}$ \\
Face-on surface density & $A_{\mathrm{HI}}(R)=v_{\infty}\left(1-\frac{1}{R^{2} / a^{2}+1}\right)$ & $A_{\max } \exp \left(\frac{(R-\mu)^{2}}{2 \sigma^{2}}\right)$ \\
& & $\rho_{0}=1.4 \mathrm{atoms} / \mathrm{cm}^{3}$ \\
& & $A \max =3 \times 10^{21} \mathrm{atoms} / \mathrm{cm}^{2}$ \\
& $\mu=4.0 \mathrm{kpc}$ \\
& $\sigma=5.3 \mathrm{kpc}$ \\
\hline \hline
\end{tabular}

Table 1. Parameters used in the toy model
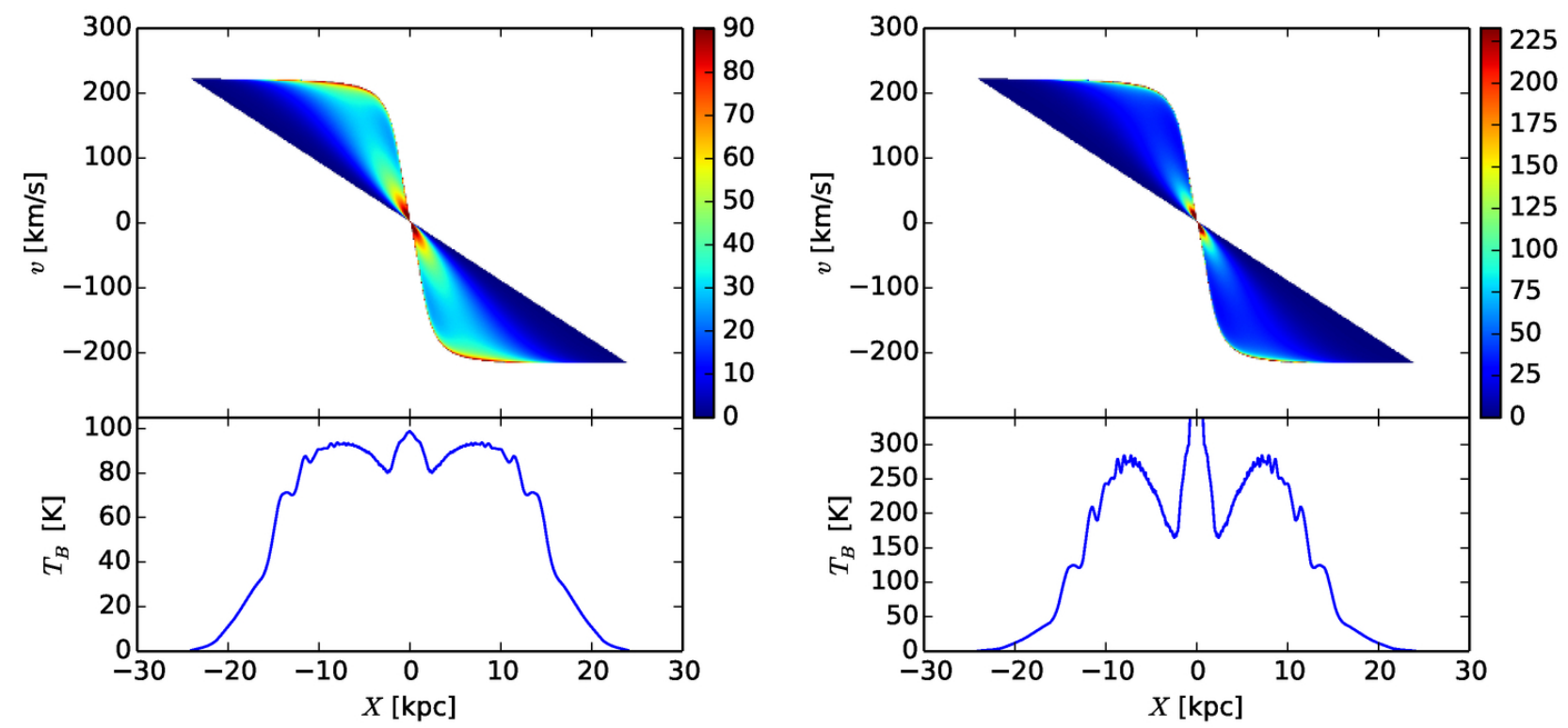

Figure 2. Position-velocity diagrams for the toy model. The vertical bars scow the scale of brightness temperature.

The left panel shows results for a self-absorption model. The right panel shows the result for an optically thin model. The lower plot in both panels shows the maximum brightness temperature at each position in the XV-diagram

is sufficiently strong, plus stronger HISA features forming distinct cloud complexes (Gibson et al. 2005, Draine 2011 Allen et al. 2012). Such cloud complexes cannot be resolved in other galaxies. Most papers on external galaxies therefore treat the HI as if it is optically thin. Evidence is however accumulating that flat-topped HI profiles characteristic of this effect are common in M31 and M33, where hydrogen mass correction factors of order 1.3 to 1.4 have been derived (Braun 2012). So what is the overall opacity of the HI in external galaxies and should we correct for it? One answer comes from O'Brien et al. (2010), who show maps of the maximum surface brightness for eight edge-on galaxies. Rather than in the conventional unit of mJy per beam, the data are presented in Kelvin. A remarkable result is found: In each galaxy, the maximum brightness along the major axis is roughly constant between 80 and $100 \mathrm{~K}$ along most of the disc. This is most clear in ESO 274-G001, of which a similar result is shown here in Figure 9 top-right. While the path-length through the disc varies drastically with position along the major axis, why can the maximum surface brightness remain so constant? O'Brien et al. (2010) speculated that this might be due to HI self-absorption. If true, this is a very important result: HI should not be treated as optically thin.

We begin by analysing a toy model of an edge-on galaxy. We roughly base our model on the observations of NGC 891 by Sancisi \& Allen (1979). NGC 891 bears close resemblance to our Galaxy (van der Kruit 1984). Because of this, we use the Solar Neighbourhood average HI density $\rho_{0}$ of 1.4 atoms $/ \mathrm{cm}^{3}$ (Mihalas \& Binney 1981. Table 9-1), as this provides a reliable estimate of the density. Rather than the value of $1 \mathrm{kpc}$ adopted by Sancisi \& Allen (1979) for the constant thickness FWHM of the disc, we use 700 pc to compensate for the revised distance of NGC 891 to $10 \mathrm{Mpc}$. The HI forms a smooth medium of constant density with height and only changes with radius, as defined through the face-on surfacedensity distribution. We use the average density $\rho_{0}$ and thickness FWHM to set the maximum face-on surface density $A$ max at $3.0 \times 10^{21}$ atoms $/ \mathrm{cm}^{3}$. The face-on surface density and rotation curve of the toy model are shown in the top 
The Shape of Dark Matter Haloes I.

\begin{tabular}{|c|c|c|c|c|c|c|c|c|}
\hline Galaxy & $\begin{array}{l}\text { RA } \\
(1)\end{array}$ & $\begin{array}{l}\text { DEC } \\
(1)\end{array}$ & $\begin{array}{l}\text { PA } \\
{\left[^{\circ}\right]} \\
(1)\end{array}$ & $\begin{array}{l}\quad[t] \\
\text { Dist } \\
{[\mathbf{M p c}]} \\
(2)\end{array}$ & $(2)$ & $\begin{array}{c}\text { Scale } \\
{[\mathrm{pc} / \text { arcsec }]}\end{array}$ & $\begin{array}{c}\text { Morphology } \\
\text { (3) }\end{array}$ & $\begin{array}{c}M_{B} \\
{\left[\begin{array}{c}\text { B-mag] } \\
(4)\end{array}\right.}\end{array}$ \\
\hline IC 2531 & $9: 59: 56.0$ & $-29: 37: 00.0$ & 74.6 & 27.2 & $\mathrm{TF}(\mathrm{a})$ & 131.7 & $\mathrm{Sc}$ & $8.835(\mathrm{~b})$ \\
\hline IC 5052 & $20: 52: 05.5$ & $-69: 12: 04.4$ & 321.2 & 5.7 & TRGB (b) & 27.4 & SBd & $9.102(\mathrm{~b})$ \\
\hline IC 5249 & $22: 47: 06.0$ & $-64: 50: 00.9$ & 14.4 & 32.1 & $\mathrm{TF}(\mathrm{a})$ & 155.4 & SBd & $12.314(\mathrm{a})$ \\
\hline ESO 115-G021 & $2: 37: 46.9$ & $-61: 20: 13.2$ & 223.2 & 4.9 & TRGB (a) & 23.8 & SBdm & $14.301(\mathrm{a})$ \\
\hline ESO 138-G014 & $17: 07: 02.2$ & $-62: 05: 20.9$ & 134.8 & 15.8 & $\mathrm{TF}(\mathrm{a})$ & 76.5 & $\mathrm{SB}(\mathrm{s}) \mathrm{d}$ & $10.663(\mathrm{~b})$ \\
\hline ESO 146-G014 & $22: 12: 59.9$ & $-62: 04: 06.3$ & 222.3 & 21.7 & $\mathrm{TF}(\mathrm{a})$ & 105.1 & SBd & - \\
\hline ESO 274-G001 & $15: 14: 14.3$ & $-46: 48: 21.0$ & 216.6 & 3.0 & TRGB (a) & 14.6 & SAd & $8.416(\mathrm{~b})$ \\
\hline UGC 7321 & $12: 17: 34.2$ & $+22: 32: 25.6$ & 8.1 & 10.0 & TRGB (c) & 48.5 & $\mathrm{Sd}$ & $10.883(\mathrm{~b})$ \\
\hline
\end{tabular}

Table 2. General properties of the galaxies in this survey. Distances are based on the Tully-Fisher relationship (TF) or the tip of the Red Giant Branch (TRGB). References: (1): This work, (2a): (Tully et al. 2008), (2b): (Radburn-Smith et al. 2011), (2c): (Matthews 2000), (3): (de Vaucouleurs et al. 1991), (4a): (Skrutskie et al. 2003) or (4b):(Jarrett et al. 2003)

row of Figure 1, with the exact parameters shown in Table 1. The disc has a maximum radius of $24 \mathrm{kpc}$. The gas in our model has no intrinsic dispersion, so all emission occurs at exactly the projected velocity $v_{\text {los }}=v_{\text {rot }} \cos (\theta)$. Here $\theta$ denotes the angle between the position of the gas $(x, y)$ inside the galaxy, and the centre of the galaxy $[\theta=\arctan (y / x)]$. The lack of intrinsic dispersion is similar to a velocity dispersion lower than one $\mathrm{km} / \mathrm{s}$. This is not realistic and would cause an overestimation of the self-absorption. To correct for this, we divide our calculated HI absorptions by a factor 8 , which is very roughly the same effect as expected due to a velocity dispersion of $8 \mathrm{~km} / \mathrm{s}$. Lewis (1984) studied 200 face-on galaxies and found the velocity dispersion to be $8 \pm 1 \mathrm{~km} / \mathrm{s}$, which is consistent with observations of the outer Galaxy (Saha et al. 2009). The results presented should thus be seen as a lower limit to the self-absorption. We do not include any form of beam smearing.

We start by tracing a single ray through the disc at a position $X$ along the major axis of nine $\mathrm{kpc}$ from the centre. The position of the ray is shown in the spider diagram in Figure 1. As the ray passes through the model, it crosses through various regions, each of which is at a particular projected velocity $v_{\text {los }}$. To illustrate this, we also show the projected velocity along the ray in Figure 1 at the right, second panel from the top. As is clear from that panel, the velocities are not evenly distributed along the ray. This means that the distance that the ray crosses -also known as the path-length- is different for each projected velocity $v_{\text {los }}$. This is demonstrated in the fifth panel of Figure 11, where we show the path-length per velocity. Near the lowest and highest velocities, the ray crosses the longest path-lengths. Not only has the velocity changed along the ray, but also the amount of atoms. While the path-lengths at the low velocities are the longest, there is also the least amount of HI there. The highest observed velocity (the terminal velocity) is near the inner radii of the model, and as such harbors the most neutral hydrogen. This is demonstrated in the sixth panel of Figure 1, where we show the amount of $N_{H_{I}}$ (in atoms $/ \mathrm{cm}^{2}$ ) per projected velocity $v$. Using Equation 1 we can subsequently convert the amount of HI to the absorption coefficient $\tau_{\nu}$ (combination of Equations 8.8 and 8.11 from Draine 2011,

$$
\tau_{\nu}=2.190 \sqrt{2 \pi} \frac{N_{H_{I}}}{10^{21}} \frac{100 \mathrm{~K}}{T_{\text {spin }}} .
$$

The spin temperature $T_{\text {spin }}$ is chosen at 100 Kelvin. The result is shown in Figure 1] where it can be seen that the absorption coefficient is roughly 0.4 throughout most of the velocity span, yet spikes to over three near the terminal velocity. The terminal velocities and thus the inner radii of the model have turned opaque. This result is similar to the (much more detailed) simulation of the Galaxy by Douglas et al. (2010), who showed that a large fraction of the Galactic plane should be optically thick. Using Equation 2 we can convert the opacity to an observed brightness (Draine 2011, Equation 7.26), which we show in the last panel of Figure 1

$T_{B}=T_{\text {spin }}\left(1-e^{-\tau_{\nu}}\right)$.

As can be seen, the observed brightness along this ray rises continuously towards the maximum of $100 \mathrm{~K}$ at the terminal velocity.

We trace rays at each position along the major axis of the disc. We then build a position-velocity (XV) diagram, such as can be seen in the top part of Figure 2. In the right panel of Figure 2 we demonstrate the equivalent result, as calculated using the optically thin conversion $\left(\tau_{\nu} \lesssim 0.1\right)$ from $N_{\mathrm{HI}}$ to $T_{B}$ given by Equation 3 (O'Brien et al. 2010),

$T_{B}=\frac{N_{H_{I}}}{1.82 \times 10^{18} d v}$.

The scales in both cases encompass the full range in intensity. Comparing both panels, we see that near the centre the diagrams are very similar, but over the rest of the diagram there is clearly a large difference between the selfabsorbing and optically thin models. In the self-absorption model, a bright bar forms near the terminal velocities. This is due to the stacking of gas at more outward positions and lower projected velocities. In the optically thin model, this gas is always visible, and any envelope tracing method will have no problem understanding it. However, the stacking is a problem in the self-absorption model, as any outer envelope tracing method will interpret the diagram as less HI at the more outward radii than is actually there.

This is further illustrated by the lower panel of both figures, where we show the maximum brightness that the ray obtains at that position. The self-absorbing model shows a plateau between 80 and $100 \mathrm{~K}$, much the same as the re- 


\begin{tabular}{|c|c|c|c|c|c|c|c|c|c|c|}
\hline \multirow[b]{2}{*}{ Galaxy } & \multicolumn{5}{|c|}{ High Resolution } & \multicolumn{5}{|c|}{ Low Resolution } \\
\hline & $\begin{array}{c}\theta_{\mathbf{F W H M}} \\
{[\operatorname{arcsec}]}\end{array}$ & $\begin{array}{c}\Delta \mathbf{v} \\
{[\mathrm{km} / \mathrm{s}]}\end{array}$ & {$\left[\frac{\sigma}{\mathbf{m J y}}\right]$} & $\begin{array}{c}\sigma \\
{[\mathbf{K}]}\end{array}$ & $\begin{array}{c}\sigma \\
{\left[\text { atoms } / \mathbf{c m}^{2}\right]}\end{array}$ & $\begin{array}{c}\theta_{\mathbf{F W H M}} \\
{[\operatorname{arcsec}]}\end{array}$ & $\begin{array}{c}\Delta \mathrm{v} \\
{[\mathrm{km} / \mathrm{s}]}\end{array}$ & {$\left[\frac{\left.\begin{array}{c}\sigma \\
\mathbf{m J y}\end{array}\right]}{\text { beam }}\right]$} & $\begin{array}{c}\sigma \\
{[\mathbf{K}]}\end{array}$ & $\begin{array}{c}\sigma \\
{\left[\text { atoms } / \mathbf{c m}^{2}\right]}\end{array}$ \\
\hline IC 2531 & 10.0 & 6.6 & 1.2 & 7.1 & $8.5 \times 10^{19}$ & 30.0 & 6.6 & 1.5 & 1.0 & $1.2 \times 10^{19}$ \\
\hline IC 5052 & 11.0 & 3.3 & 1.4 & 6.8 & $4.1 \times 10^{19}$ & 30.0 & 3.3 & 1.7 & 1.1 & $6.6 \times 10^{18}$ \\
\hline IC 5249 & 8.0 & 6.6 & 1.1 & 10.8 & $1.3 \times 10^{20}$ & 30.0 & 6.6 & 1.9 & 1.3 & $1.5 \times 10^{19}$ \\
\hline ESO 115-G021 & 10.6 & 3.3 & 1.2 & 6.6 & $3.9 \times 10^{19}$ & 30.0 & 3.3 & 1.4 & 0.9 & $5.5 \times 10^{18}$ \\
\hline ESO 138-G014 & 11.8 & 6.6 & 1.7 & 7.3 & $8.7 \times 10^{19}$ & 30.0 & 6.6 & 2.3 & 1.6 & $1.9 \times 10^{19}$ \\
\hline ESO 146-G014 & 8.6 & 6.6 & 1.2 & 9.7 & $1.2 \times 10^{20}$ & 30.0 & 6.6 & 1.7 & 1.1 & $1.4 \times 10^{19}$ \\
\hline ESO 274-G001 & 12.2 & 3.3 & 1.5 & 6.2 & $3.7 \times 10^{19}$ & 30.0 & 3.3 & 1.8 & 1.2 & $7.2 \times 10^{18}$ \\
\hline UGC 7321 & 13.8 & 5.2 & 0.3 & 1.0 & $9.8 \times 10^{18}$ & 30.0 & 5.2 & 0.5 & 0.4 & $3.4 \times 10^{18}$ \\
\hline
\end{tabular}

Table 3. The global properties as measured from both the high and low-resolution HI cubes. Shown are the size of the beam $\theta_{\mathrm{FWHM}}$, the width and stepping of the velocity channels $\Delta v$ and the one-sigma background noise expressed in various units.

sults by O'Brien et al. (2010). In contrast, the optically thin model does not show such a plateau at al The observed brightness temperature rises well above $200 \mathrm{~K}$ in the inner part. Larger regions with brightness levels well above $100 \mathrm{~K}$ are never observed in reality. The maximum brightness peak in O'Brien et al. (2010) is still below $200 \mathrm{~K}$. Even in the high-resolution observations available by studying our own Galaxy, temperatures above $150 \mathrm{~K}$ are uncommon (Taylor et al. 2003, Douglas et al. 2010).

So what is the consequence of this? As demonstrated by this toy model, the presence of a maximum brightness plateau along most of the major axis, as well as the limited brightness, are both strong indications of HI self-absorption. We conclude that any subsequent modelling will need to take into account this effect, or risk underestimating the HI content of the galaxies.

\section{SAMPLE \& OBSERVATIONS}

Our sample is the same as that of O'Brien et al. (2010). Criteria for the galaxies were that they lie in the southern sky, are close to edge-on $(a / b \geqslant 10)$, Galactic latitude $\|b\| \geqslant$ $10^{\circ}$ as to avoid optical and infrared extinction by the Galaxy, relatively bulge-less (Hubble type Scd-Sd), nearby enough to be able to resolve the flare $(d \leqslant 30 \mathrm{Mpc})$ and with a minimal integrated flux of $15 \mathrm{Jy} \mathrm{km} / \mathrm{s}$. This led to a sample of five edge-on galaxies. Two other galaxies were added for various reasons. UGC 7321 was added due to the availability of very high quality archival data, from the work by Uson \& Matthews (2003). ESO 274-G001, despite its low Galactic latitude of $9.3^{\circ}$, was included for its exceptional proximity. IC 2531, a box/peanut bulge, barred galaxy was included as a test of halo shapes under different types of mass scales and stages of secular evolution. We list the global properties of the sample in Table 2 We refer the reader to Paper IV for more optical and near-infrared images of these galaxies.

The results presented here come from both archived data as well as new observations. Almost all were observed using the Australian Telescope Compact Array (ATCA) near Narrabri, Australia. The only exception is UGC 7321, which was based on archived observations from the Very Large Array (VLA). O'Brien et al. (2010) also included
ATCA observations for this galaxy, but we found that this limited 4 hour observation reduced the overall quality of the cube. Most of the observations came from previous archived observations by various authors over a range of years, going back to 1993. A significant number of the observations was already used by O'Brien et al. (2010). A major new set of observations came as part of the work undertaken by The Local Volume HI Survey (LVHIS) group (Koribalski 2008). For ESO 138-G014 and ESO 274-G001, we have taken new observations using the ATCA. The various observations and configurations are listed in Table ??. In total, we have collected more than 132 hours of additional observation-time compared to O'Brien et al. (2010).

\section{REDUCTIONS}

\subsection{Calibration \& Imaging}

The calibration and imaging of the data has been done in MIRIAD (Sault et al. 1995). All subsequent analysis was done in PYTHON. All archived data from the ATCA was take in the $\mathrm{XX}$ and $\mathrm{YY}$ polarizations. The new observations also include the XY and YX polarizations. We made use of the pre-calibrated data from O'Brien et al. (2010), although we have re-reduced parts where we believed improvements could be made.

The data were first imported and split into the various observations, excluding any telescope shadowing. We then inspected all data for RFI and flagged where necessary. The primary and secondary calibrator were then used to calculate the gains and band-passes. This was then applied to the main target. The continuum was then subtracted from the UV data.

The imaging was done using a three-pass strategy, similar to O'Brien et al. (2010). Since the exact pointing sometimes varied between observations, we used the MIRIAD "joint approach" to invert the data in mosaic mode. The data were imaged in Stokes II, which is a special case of Stokes I, under the assumption of an unpolarised source ${ }^{2}$ A robust parameter of 0.4 was used to provide a good balance between beam-size and side-lobes.

In the first pass, we cleaned the full cube, using the
1 Note that the bumps in the profile are due to the finite numerical resolution
2 From the Miriad user manual at http://www.atnf.csiro.au/ computing/software/miriad/userguide/node108.html 
maximum-entropy MOSMEM task ${ }^{3}$ This was then restored, which resulted in an estimate for the major and minor axis of the beam. This was taken as the basis for the second pass. Here we used a square cell-size of half the full-width halfmaximum (FWHM) of the major axis beam. The central quadrant was again cleaned and restored, using a circular beam with the FWHM of the major axis of the beam from the previous pass. The restored cube was smoothed to twice the FWHM and the standard deviation $\sigma$ of the background noise determined. We defined a regions mask in the cube where the signal strength was more than double the noise. In the third pass, the data raw cube was again cleaned with MOSMEM, this time only using the new regions mask.

We also produced low-resolution cubes with a beam of $30^{\prime \prime}$ to search for any faint, extended component. This was done by tapering the Fourier transformation to $30^{\prime \prime}$. The clean was then based on the same regions as the highresolution cube. In some cases we found the regions too small for the low-resolution beam, such that parts of the galaxy were cut off. The regions were then widened and both the high- and low-resolution cubes were again cleaned and restored using these new regions.

The VLA data for UGC 7321 consisted of very small band-passes, such that there was no possibility to perform a continuum subtraction in the UV-plane. The continuum was therefore subtracted in the image plane. The above strategy led to a beam of 15 arcsec for UGC 7321. We have applied an additional side-lobe suppression of 700 arcsec to the beam, which led to the smaller beam as reported here.

For IC 2531, IC 5249, ESO 138-G014 and ESO 146G014, the channel-width and spacing was originally $3.298 \mathrm{~km} / \mathrm{s}$. However, since these galaxies are the furthest, they also have the highest pc/arcsec scales (see Table 3), which meant that the original beams were not fully resolving the inner disc. We have therefore doubled the channelwidth and spacing, lowering the noise and thus enabling us to decrease the size of the beam, such that all have a FWHM beam of $\sim 1 \mathrm{kpc}$. This failed for IC 2531, where the effective noise was too high for any practical purpose. The beam for that galaxy has now been set at $1.3 \mathrm{kpc}$. A similar problem occurred for IC 5249, where we now have set the beam to a FWHM of $1.25 \mathrm{kpc}$.

\subsection{Analysis}

The noise in a mosaicked cube varies with position. It is therefore important to measure the noise as close to the galaxy as possible, while avoiding any contamination from the galaxy. The region-mask offered a good tool for this. We combined all region-masks into a single master-mask, which defined the maximum extent of the region-masks. The noise was then measured on the pixels in every channel that were part of the master mask, but were outside the specific region of that channel. We show the noise estimates in Table 3 . Following the results from Section 3, we present all results in Kelvin, as this is the most natural unit in which we can

3 There is an ongoing debate on the merits of using clean versus maximum entropy. We have chosen the maximum entropy method, as the MIRIAD user guide recommends this as the superior method to use, in particular for extended emission. identify self-absorption. We convert from flux density $S$ in Jy/beam to surface brightness temperature $T_{B}$ in Kelvin using Equation 4 and to column densities $N_{\mathrm{HI}}$ in atoms $/ \mathrm{cm}^{2}$ using the optically thin Equation 3 .

$T_{B}=\frac{\lambda^{2} S}{2 k_{B} \Omega}=\frac{606000 S}{\theta^{2}}$.

The central position and position angle (PA) were derived from the high-resolution cubes. First, the cube was clipped below $30 \mathrm{~K}$, as the lower intensity regions were often found to be asymmetrical. A clear example of this asymmetry can be seen in IC 5052 (see Figure 4 bottom-left). We then created a moment-zero image of the cube. The centre of the galaxy was found as the minimal $\chi^{2}$ of the difference between the image and its $180^{\circ}$ rotated counterpart, as trialed over a wide range of positions. The position angle was then found by rotating the galaxy over a range of possible angles around this central position. The lowest $\chi^{2}$ between the rotated images and its upside-down flipped counterpart gave the position angle. We define the position angle such that the receding side always becomes the left side, when rotating the image. The results for this are accurate to within 0.1 pixel and 0.1 degree and are listed in Table 2 . These positions will only serve as first-guess positions for the kinematic fitting in Paper III, and are thus not particularly important.

Both the high and low-resolution cubes were rotated, using the central position and PA, such that the major axis of the galaxy was aligned with the horizontal axis of the image. We created zeroth moment maps for both rotated cubes, using two times the measured background noise $\sigma$ for clipping. These cubes are shown in the bottom-left panels of Figures 3-10. The high-resolution zeroth-moment map was also used to create contour levels on top of the Digital Sky Survey (DSS) J-band image of that region. These are shown in the top-left panels of Figures 3-10.

As shown in Section 3, it is also interesting to compute the maximum-brightness-temperature maps of the galaxies, as they are instrumental in detecting any potential optical depth issues. The cube was converted from intensities $I$ in $\mathrm{mJy} /$ beam to brightness temperatures $T_{B}$ in $\mathrm{K}$, using Equation 4 . We created a maximum brightness temperature map by selecting the maximum temperature along the velocity axis $v$ for each position $(X, z)$. These are shown in the top-right panels of Figures 3-10. The middle plot shows the maximum temperature along the above-the-plane height $z$ for each position $(X, v)$. This creates an equivalent to a position-velocity diagram, only here it shows the maximum brightness-temperature regardless of height $z$. The lower panel takes the maximum brightness-temperature along both $v$ and $z$, and infers the corresponding optical depth, based on assumed spin temperature of 150, 200 and 300 Kelvin. We chose the values to lie close to the mean observed values for the cold neutral medium (CNM) (Dickey et al. 2009). As such, it forms a diagnostic tool for potential self-absorption issues. We calculated the opacity $\tau_{\nu}$ using Equation 5] which is the inverse of Equation 2

$\tau_{\nu}=-\ln \left(1-T_{B} / T_{\text {spin }}\right)$.

The integrated position-velocity (XV) diagrams are shown in the bottom-right panels of Figures 3-10. These have been created by integrating the rotated high-resolution cubes along their minor axis and converting them to bright- 


\begin{tabular}{lcccccc} 
Galaxy & $\begin{array}{c}\text { vsys } \\
{[\mathbf{k m} / \mathbf{s}]}\end{array}$ & $\begin{array}{c}\mathbf{v m a x} \\
{[\mathbf{k m} / \mathbf{s}]}\end{array}$ & $\begin{array}{c}W_{20} \\
{[\mathbf{k m} / \mathbf{s}]}\end{array}$ & $\begin{array}{c}W_{50} \\
{[\mathbf{k m} / \mathbf{s}]}\end{array}$ & $\begin{array}{c}\mathbf{F I} \\
{[\mathbf{J y} \mathbf{~ k m} / \mathbf{s}]}\end{array}$ & $\begin{array}{c}M_{\mathbf{H I}} \\
{\left[\mathbf{M}_{\odot}\right]}\end{array}$ \\
\hline \hline IC 2531 & 2463.8 & 260.5 & 484.8 & 468.3 & 41.7 & $7.3 \times 10^{9}$ \\
IC 5052 & 596.7 & 114.4 & 197.9 & 181.4 & 117.7 & $8.9 \times 10^{8}$ \\
IC 5249 & 2351.7 & 131.9 & 234.2 & 217.7 & 23.2 & $5.6 \times 10^{9}$ \\
ESO 115-G021 & 514.2 & 85.1 & 141.8 & 125.3 & 109.0 & $6.2 \times 10^{8}$ \\
ESO 138-G014 & 1492.3 & 130.9 & 237.5 & 227.6 & 48.9 & $2.9 \times 10^{9}$ \\
ESO 146-G014 & 1678.8 & 84.1 & 148.4 & 128.6 & 17.5 & $1.9 \times 10^{9}$ \\
ESO 274-G001 & 523.6 & 103.9 & 181.4 & 168.2 & 150.1 & $3.2 \times 10^{8}$ \\
UGC 7321 & 409.3 & 128.8 & 237.0 & 221.6 & 41.7 & $9.8 \times 10^{8}$ \\
\hline \hline
\end{tabular}

Table 4. Spectral properties of the galaxies

ness temperatures. The right-hand panels show the integrated spectra $S(v)$, which were created by integrating the XV-diagrams over the major axis. Similarly, we show the brightness as function of major-axis position in the lower plots, by integrating the XV diagrams over $v$.

The width of the profile at the $20 \%$ and $50 \%$ maximum intensity levels, $W 20$ and $W 50$, were measured directly from $S(v)$. These were based on the low-resolution cubes, which were masked using the regions files. The integrated flux FI was also measured in this cube. We make no use of clipping, in contrast to O'Brien et al. (2010), as we wish to include all faint emission. The total optically thin $\mathrm{HI}$ mass, $M_{\mathrm{HI}}$, was derived using

$M_{\mathrm{HI}}=2.343 \cdot 10^{5} \times D^{2} \times \mathrm{FI}$,

where the adopted distance $D$ is in Mpc as shown in Table 2 (Draine 2011). This value will represent only a lower limit to the true HI mass. The derived values for FI, $M_{\mathrm{HI}}, W 20$ and $W 50$ are shown in Table 4

The systematic velocity vsys was measured from the $\mathrm{XV}$-diagram in a method similar to the position finding. For a wide range of possible systemic velocities $v_{s y s}$, the $\mathrm{XV}$-diagram was rotated $180^{\circ}$ around that velocity. The rotated XV-diagram was subtracted from the original, with the lowest $\chi^{2}$ was adopted for vsys. The maximum velocity vmax was calculated by locating the furthest channel from vsys that still contained flux above two times the $\sigma$ background noise level. The results for vsys and vmax are also shown in Table 4 The channel-maps are presented in online Appendix ??.

\section{RESULTS}

\subsection{IC 2531}

Galaxy IC 2531 is the only Sc galaxy in our sample. The classification is different from that of Bureau \& Freeman (1999) and O'Brien et al. (2010), where the galaxy has been classified as a Sb galaxy. The classification of Sb occurs first in Bureau \& Freeman (1999), who note that this classification was not extremely accurate. It has the largest gas mass of the sample, and has a maximum rotation velocity vmax which is twice as high as the rest of the sample (Table 4). The HI layer is remarkably flat, with the disc seemingly having a smaller scale-height than the stellar disc (Figure 3 top-left). All of this is supports an Sc classification. It is true that the bulge of IC 2531 is not very weak as in many Sc's, so that a Sbc classification cannot be ruled out. In any case, we believe the evidence to point at a later classification than Sb.

Bureau et al. (2006) have shown that at K-band IC/,2531 shows signatures of a peaunut-shaped or boxy spheroid with a X-shape structure in the unsharp masked image of the bulge, which would be associated with a bar (see e.g. Athanassoula 2016, for a recent review). Based on the double horns in the integrated profile, we would expect the galaxy to be symmetric (Figure 3 bottom-right). The XVdiagram itself is however asymmetric. Interestingly, HIPASS also reports a more asymmetric profile. The asymmetry is also present in the analysis of the galaxy in O'Brien et al. (2010b their Figure 15), where the two sides of the derived surface density can be seen to deviate strongly.

The XV-diagram shows a so-called "figure 8"-pattern, for which Bureau \& Freeman (1997) suggest that it is due to an extended weak bar. This bar did not show up in in the $\mathrm{H} \alpha$ analysis of Bureau \& Freeman (1999), although they suffered from background problems for this galaxy. This lack of detection was not fully unexpected, as Bureau \& Freeman (1997) already noted that the figure extended over a very long range. Bureau \& Freeman speculated that the figure might also be due to a warp, density ring or a spiral arm. Ann \& Park (2006) confirmed the presence of a warp in this galaxy. We also think it more likely that this pattern is associated with the warp and does not represent a bar. The warp is visible in Figure 3 bottom-left. The K-band observations mentioned above do show evidence for a central bar, but this this bar does not appear to extend over much more than the central hole in the HI distribution.

The XV-diagram is also asymmetric in the rotation curve. The receding side shows a slowly increasing rotation curve, while the approaching side show a sharp increase followed by a flat rotation.

The maximum surface brightness temperature map (Figure 3 top-right) shows a strong central peak and some additional peaks. There does not appear to be symmetry in the distribution of these peaks.

\section{$6.2 \quad$ IC 5052}

IC 5052 is a nearby SBd galaxy. The bulge is clearly visible in Figure 4 top-left. We find a previous unreported, line-ofsight warp in the outskirts of the galaxy. This is particularly clear in the zeroth-moment map of the low resolution cube (Figure 4 bottom-left). Further modelling will be required to confirm this as a true warp, although this is beyond the scope of this project. For now, we note that the channel 


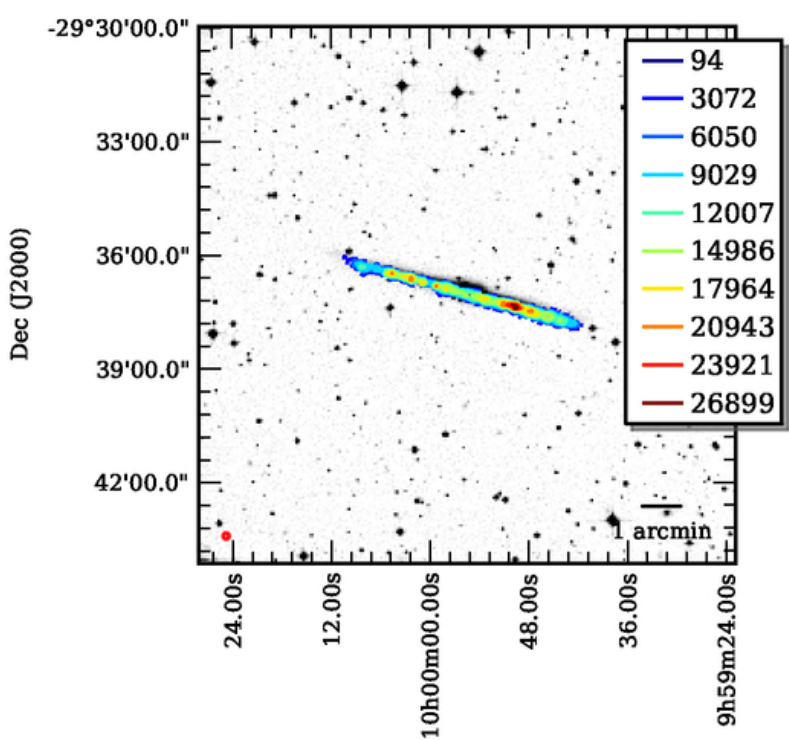

RA (J2000)

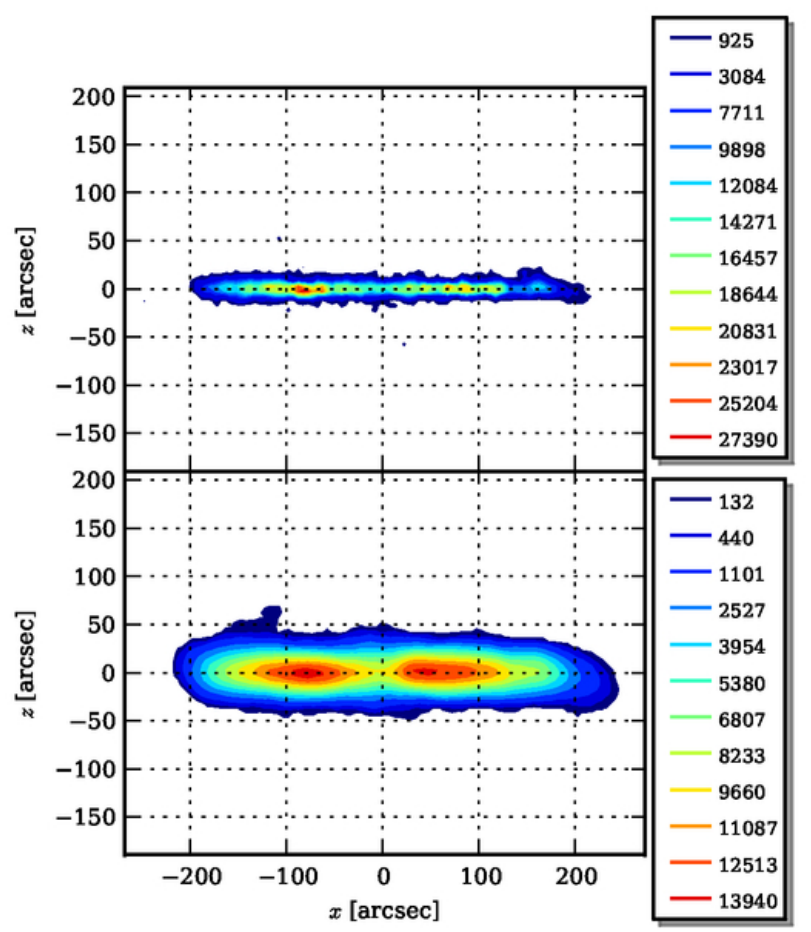

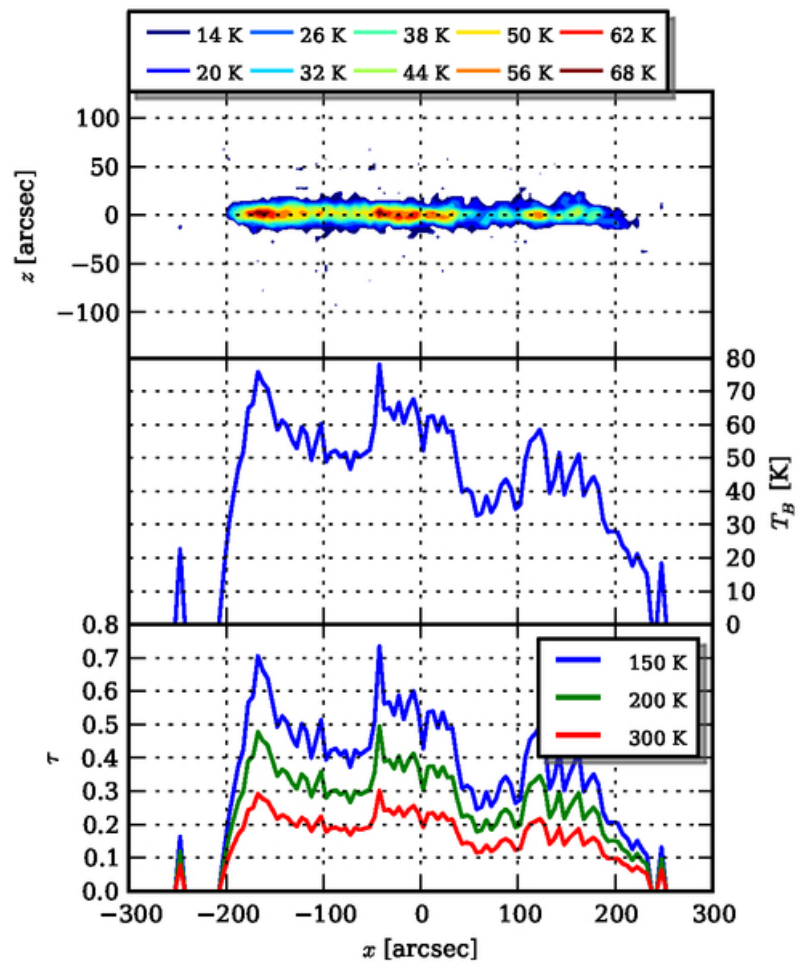

[Jy]

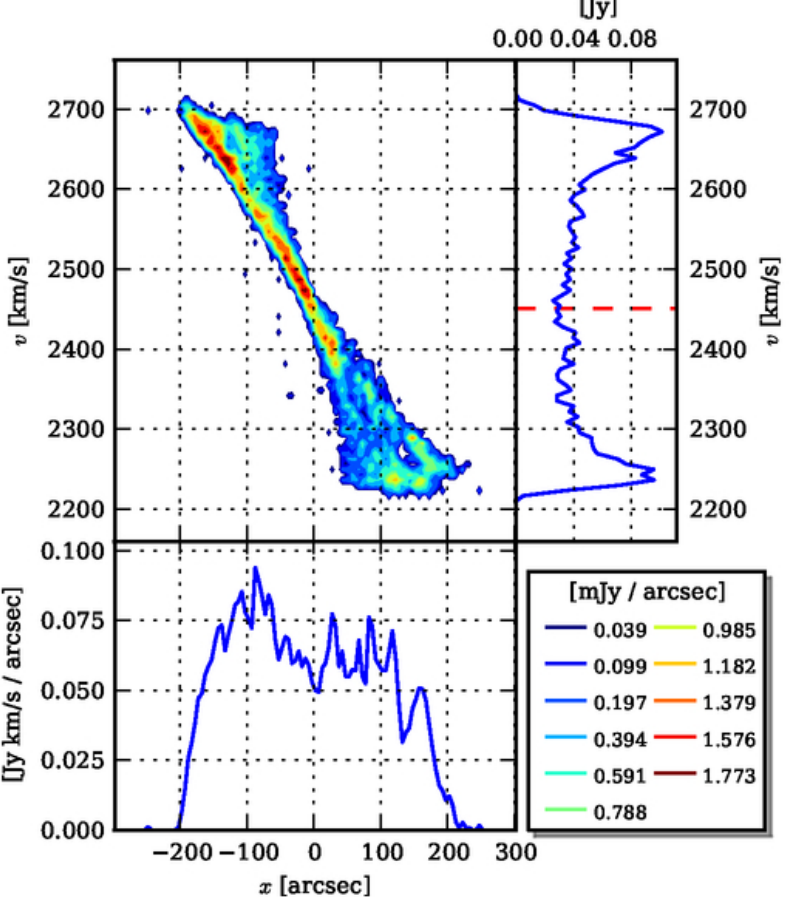

Figure 3. Overview of the neutral hydrogen contents of IC 2531. Top-left: DSS1 overlaid with contours from the HI moment 0 map in $[\mathrm{K} /(\mathrm{km} / \mathrm{s})]$. Top-right: Top panel shows the maximum temperature along $v$. The middle panel shows the maximum temperature along $z$ with the same scale. The lower plot maximum temperature along $v$ and $z$ and shows the inferred self-absorption for that position, assuming a spin temperature of $125 \mathrm{~K}$. Bottom-left: Top panel shows the moment 0 for the high-resolution cube in $[\mathrm{K} /(\mathrm{km} / \mathrm{s})]$. Lower plot shows the moment 0 for the low-resolution cube. Bottom-left: Left panel shows the PV-diagram. Right-top panel shows the integrated flux per velocity. The red dashed line shows vsys. The lower plot shows the integrated flux per position along the major axis. 


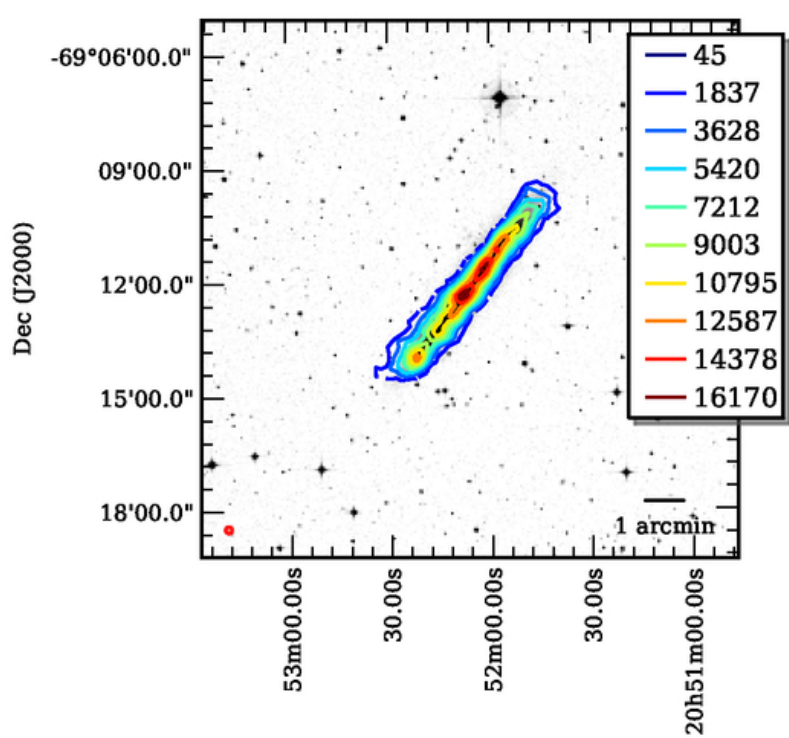

RA (J2000)
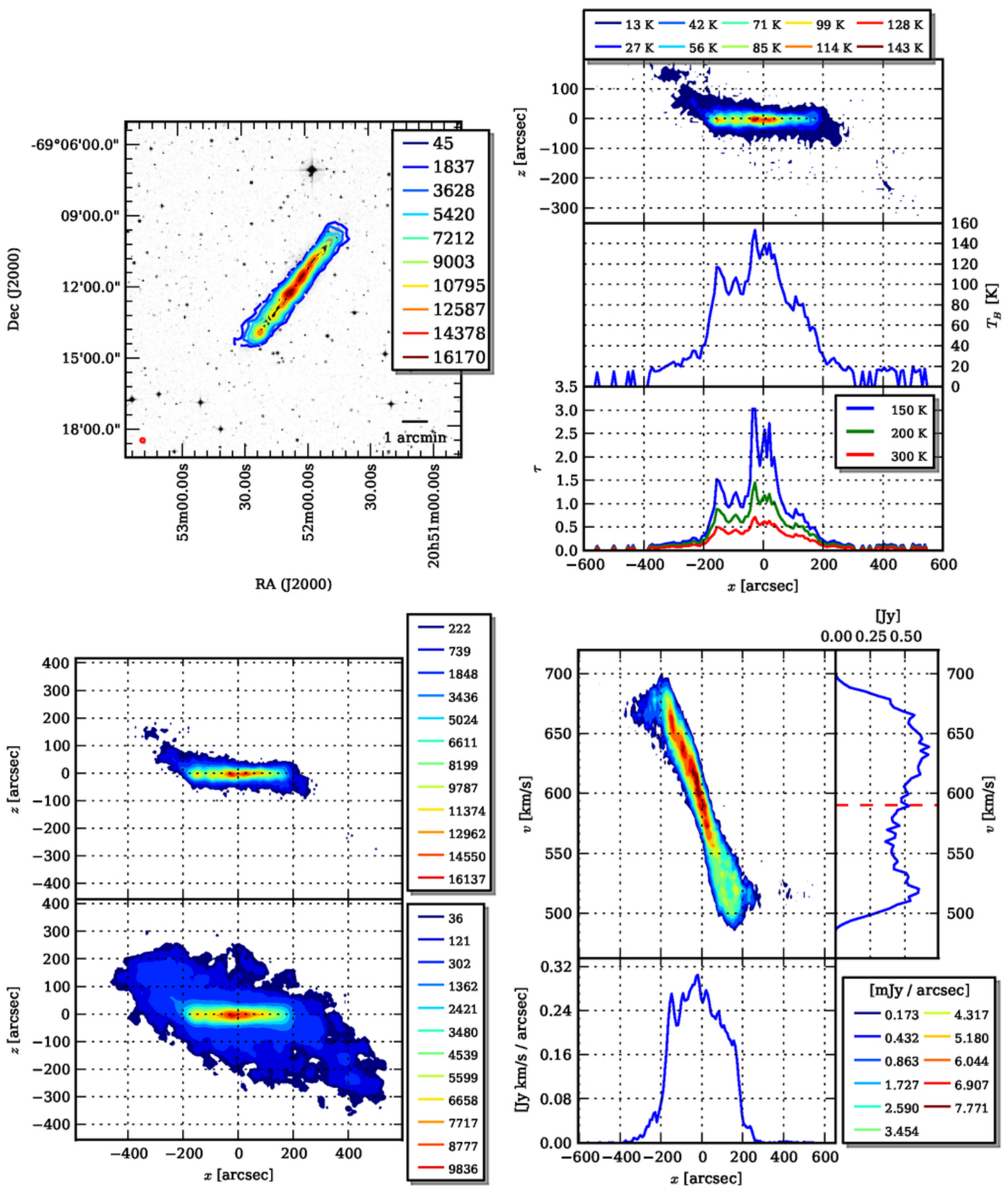

[Jy]

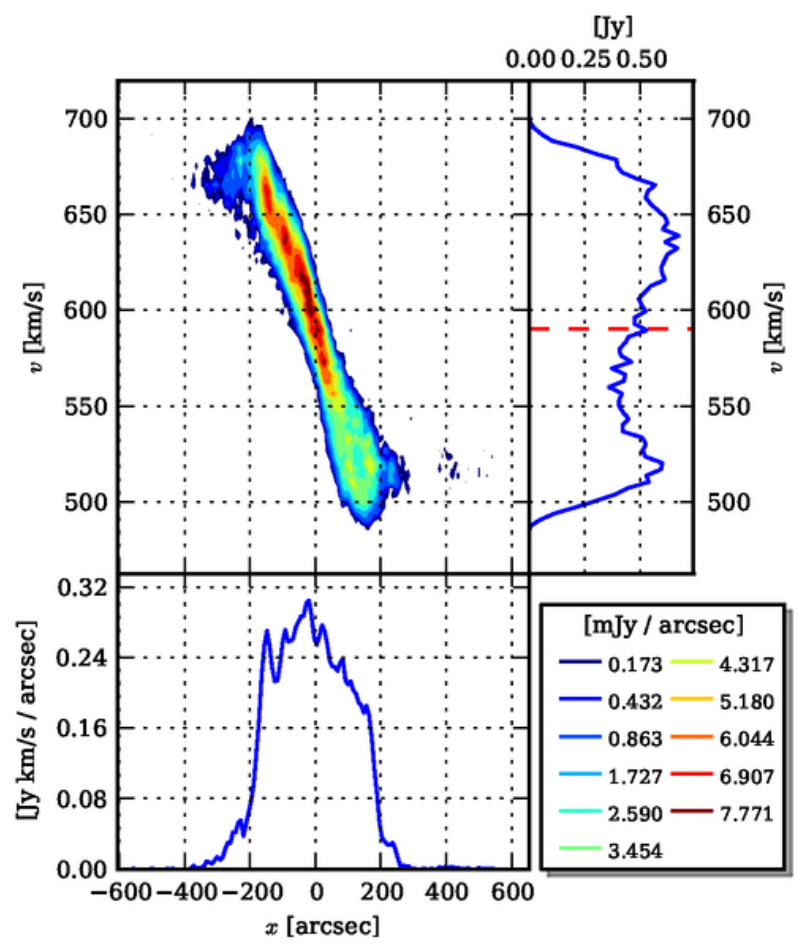

Figure 4. Overview of the neutral hydrogen contents of IC 5052. Top-left: DSS1 overlaid with contours from the HI moment 0 map in $[\mathrm{K} /(\mathrm{km} / \mathrm{s})]$. Top-right: Top panel shows the maximum temperature along $v$. The middle panel shows the maximum temperature along $z$ with the same scale. The lower plot maximum temperature along $v$ and $z$ and shows the inferred self-absorption for that position, assuming a spin temperature of $125 \mathrm{~K}$. Bottom-left: Top panel shows the moment 0 for the high-resolution cube in [K/(km/s)]. Lower plot shows the moment 0 for the low-resolution cube. Bottom-left: Left panel shows the PV-diagram. Right-top panel shows the integrated flux per velocity. The red dashed line shows vsys. The lower plot shows the integrated flux per position along the major axis. 


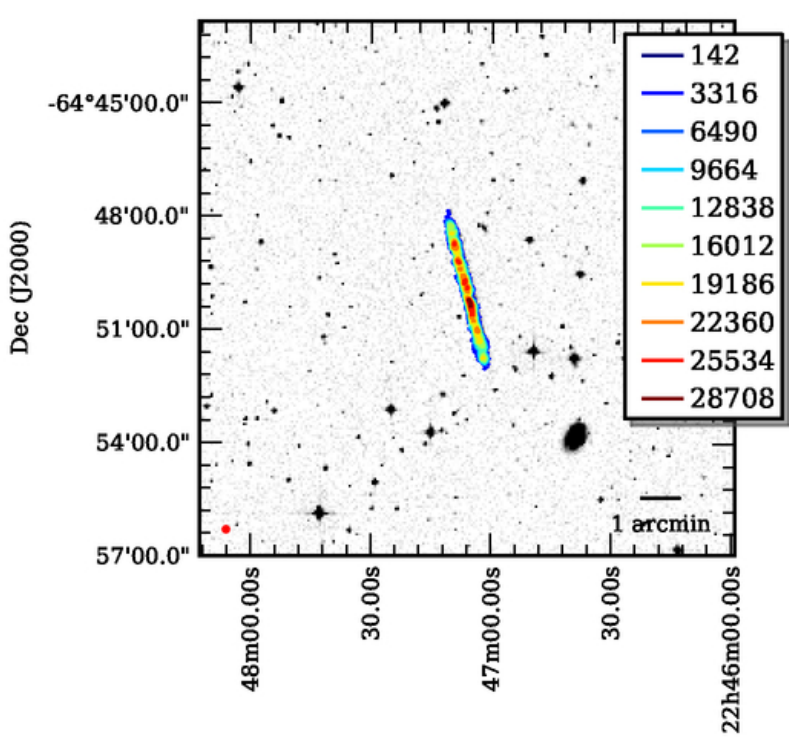

RA (J2000)

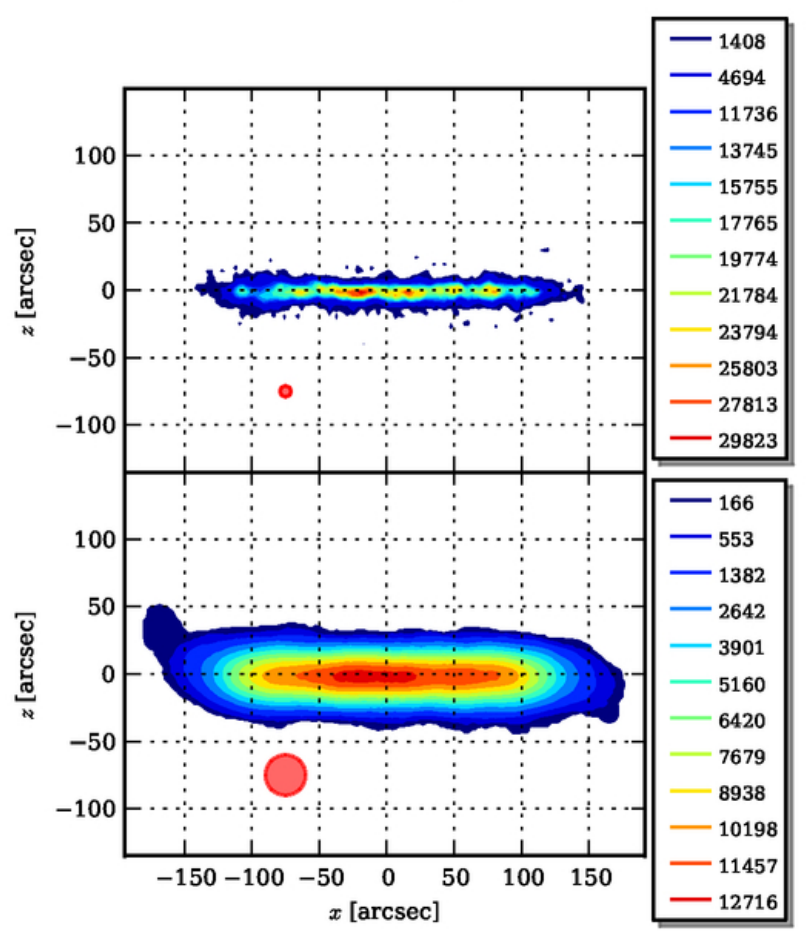

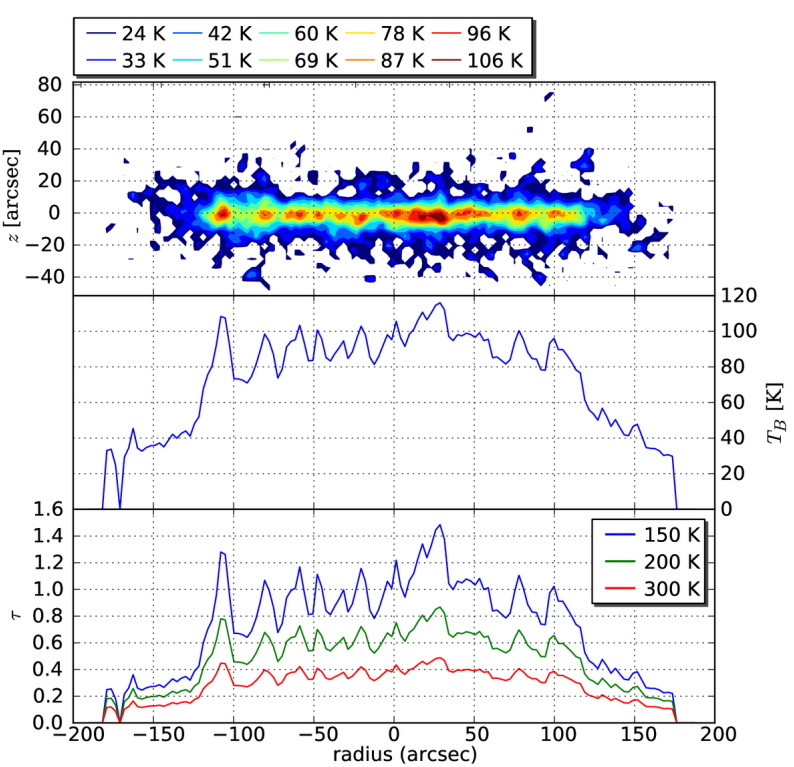

[Jy]

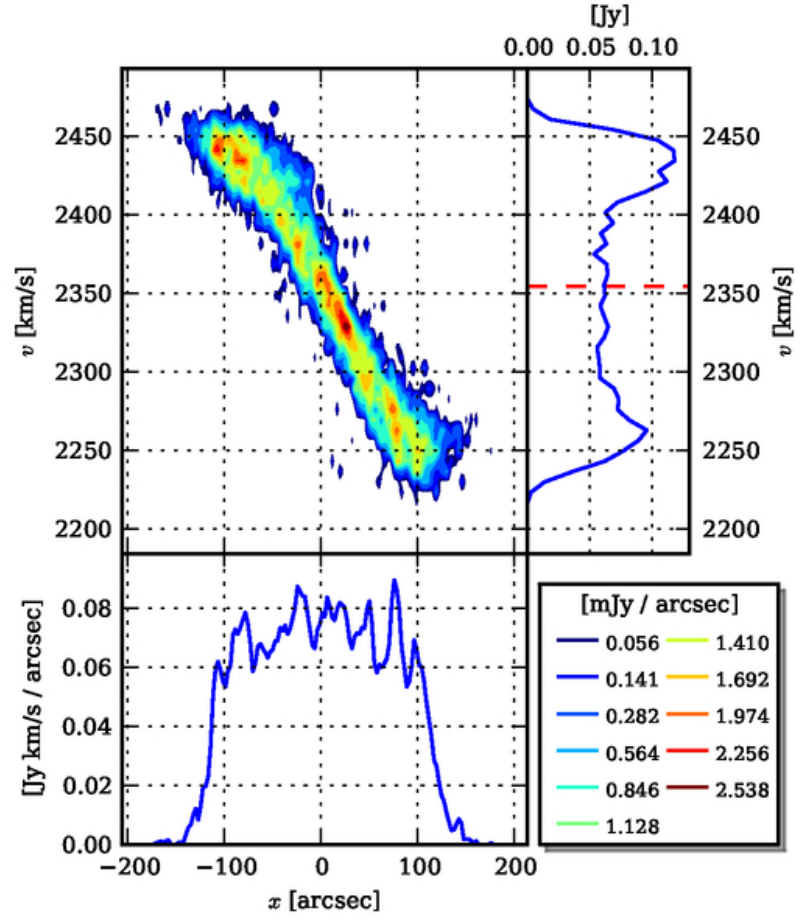

Figure 5. Overview of the neutral hydrogen contents of IC 5249. Top-left: DSS1 overlaid with contours from the HI moment 0 map in $[\mathrm{K} /(\mathrm{km} / \mathrm{s})]$. Top-right: Top panel shows the maximum temperature along $v$. The middle panel shows the maximum temperature along $z$ with the same scale. The lower plot maximum temperature along $v$ and $z$ and shows the inferred self-absorption for that position, assuming a spin temperature of $125 \mathrm{~K}$. Bottom-left: Top panel shows the moment 0 for the high-resolution cube in [K/(km/s)]. Lower plot shows the moment 0 for the low-resolution cube. Bottom-left: Left panel shows the PV-diagram. Right-top panel shows the integrated flux per velocity. The red dashed line shows vsys. The lower plot shows the integrated flux per position along the major axis.

maps, seen in Figures ??, ??, ?? in online Appendix B, are very similar to the fitted model of the line-of-sight warp in ESO 123-G13 (Gentile et al. 2003). The warp appears to be symmetric in pitch angle on either side of the galaxy, although it is more extended towards the lower-left side. We have checked various optical and infrared images, but we can find no optical counterpart to the warp.

The XV-diagram of this galaxy (Figure 4 bottom-right) shows signs of this warp beyond 250 arcseconds. The main part of the profile looks like a solid-body rotator. The highand low velocity sides are asymmetric, which is also clear from the integrated profile. The shape of the spectrum does agrees well with the profile from HIPASS.

The maximum surface brightness temperature profile of Figure 4 top-left shows a plateau around 80-100 K for most of the inner part of the galaxy. Only the central part 
shows a higher temperature, rising to a maximum of $140 \mathrm{~K}$. This central over-density is also visible in the zeroth-moment maps 4 bottom-left). O'Brien et al. (2010) identify this as a star-forming region.

\subsection{IC 5249}

IC 5249 is the most distant galaxy in our sample, at a distance of $32 \mathrm{Mpc}$. The galaxy is only five arcminutes wide. The maximum $\mathrm{S} / \mathrm{N}$ of the cube has not been improved since O'Brien et al. (2010), and is still around ten.

The maximum temperatures exhibit a plateau between 80 and $100 \mathrm{~K}$, over the radius -100 and 100 arcseconds, along most of the galaxy (Figure 5 top-right). Another plateau is visible in the integrated flux along the major axis 5 bottomright), wherein over that same region the intensity is seen to hover around $0.06 \mathrm{Jy} \mathrm{km} / \mathrm{s} /$ arcsec. The XV-diagram itself is slightly asymmetric, with the higher velocity side having more HI gas than the low velocity side.

The rotation curve has been previously analysed by van der Kruit et al. (2001) using HI and $\mathrm{H} \alpha$. They find the galaxy to exhibit a steep rising rotation curve, after which at 1.0 arcmin it flattens out at $v_{\max } \approx 100 \mathrm{~km} / \mathrm{s}$.

There is a faint indication of a warp, visible in the lower plot in Figure 5 bottom-left.

\subsection{ESO 115-G021}

ESO 115-G021 is a nearby SBdm galaxy at a distance of only $4.9 \mathrm{Mpc}$. A lot of data was available, totaling to 83.5 hours of observation time. Due to this, it is a very well resolved galaxy. The plane of the galaxy is resolved at over 80 beams and the galaxy covers 10 beams in height (see Figure6 topleft).

The derived spectrum is more symmetric than the HIPASS spectrum, which has a larger peak at the highvelocity side. There is an external gas cloud at 02:36:59 61:18:41 visible in multiple channels. It shows in both the high and low resolution cubes (Figure 6 bottom-left) at position (180", 300"). In the XV-diagram (Figure 6 bottomright) it is visible as the small upward peak between 500 and $515 \mathrm{~km} / \mathrm{s}$. This close proximity to the galaxy in both velocity and distance indicates the gas is associated with the galaxy. The gas cloud has an HI mass of $3.75 \times 10^{5} \mathrm{M}_{\odot}$. The cloud is invisible in the DSS, I and V bands (see Paper IV). The DSS image goes down to a limiting magnitude of 22.5 R-mag/ $\operatorname{arcsec}^{2}$, which corresponds to an apparent magnitude of 15.5 R-mag for the area of $\sim 600 \operatorname{arcsec}^{2}$ that the cloud covers. This is equal to a solar luminosity of $\sim 2.84 \times 10^{4} \mathrm{~L}_{\mathrm{R} \odot}$. The cloud thus has a mass-to-light ratio $M_{\mathrm{HI}} / L_{\mathrm{R}} \geqslant 13.3$.

The XV-diagram (Figure 6 bottom-right) shows an asymmetry, with the high velocity side being more extended than the low velocity side. This is most likely due to the warp, which is visible in the zeroth-moment maps (Figure 6 bottom-left). The main component of the XV-diagram shows solid body rotation.

The maximum temperature map (Figure 6 bottomright) shows that this warp has a surface brightness of 20$30 \mathrm{~K}$. The more stable inner parts of the disc show a maximum temperature plateau around $80-90 \mathrm{~K}$, with the strong exception of the region between $0 "$ and +50 ", where there is a rise to a maximum of $140 \mathrm{~K}$. This region corresponds to a bright region of the DSS image.

\subsection{ESO 138-G014}

Galaxy ESO 138-G014 has the least observing time of all the galaxies in our sample. Nevertheless, the galaxy is well resolved by the telescope. The minor axis of the galaxy is covered by roughly six beams.

The XV-diagram (7 bottom-right) is asymmetric, with the receding side showing an extended component. This is associated with a warp, as visible in Figure 7 bottom-left. No optical counterpart to this warp is known (Sánchez-Saavedra et al. 2003).

The maximum brightness temperature map forms a plateau throughout most of the galaxy, averaging between 80 and $100 \mathrm{~K}$ (Figure 7 bottom-right). The only exception is at the left side, where a peak of $140 \mathrm{~K}$ is attained. This corresponds to the bright region in the south-west region in the DSS image (Figure 7 top-left).

\subsection{ESO 146-G014}

Galaxy ESO 146-G014 is the faintest galaxy in our sample. The moment-zero maps (Figure 8 bottom-right) show that the galaxy does not appear to have a fixed plane. Instead it looks slightly bent. This is most likely due a strong warp, which can also be seen in the visible in the DSS J-band image (Figure 8 top-left).

The XV-diagram (Figure 8 top-right) looks symmetrical, although the integrated spectrum is more asymmetric than the HIPASS spectrum, with a stronger peak at the lowvelocity side. Overall, the galaxy appears to exhibit solidbody rotation.

The maximum surface brightness temperatures appear to form a plateau over the major part of the galaxy, averaging around $90 \mathrm{~K}$ (Figure 8 bottom-right). On the left-hand side of the galaxy, this maximum drops relatively slowly towards the warp.

\subsection{ESO 274-G001}

ESO 274-G001 is a SAd galaxy. At only $3.02 \mathrm{Mpc}$, it is the closest galaxy in our sample. It is also has the lowest HI mass of the galaxies in our sample (Table 4). Due to its proximity, it is still the largest galaxy in terms of angular size, spanning 13 arcminutes in width. In height, it is also very well resolved, covering at least 80 arcseconds.

The central hole in the zeroth-moment image (Figure 9 top-left) is an artifact, due to the removal of a continuum radio source at this position. It has a flux density of $48.65 \mathrm{mJy}$ and coincides with (or at least is very close) to the symmetry center of both the optical image, the HI distribution and the positon-velocity diagram. The Parkes single-dish 6-cm flux density is about $50 \mathrm{mJy}$ (Wright et al. 1994), so the source appear to be non-thermal. The most likely explanation is an active galactic nucleus (AGN). The incidence of AGN in low luminosity galaxies is probably low; Condon et al. (2002) indicate that the AGN phenomenon is limited to large to $\mathrm{E}$ 


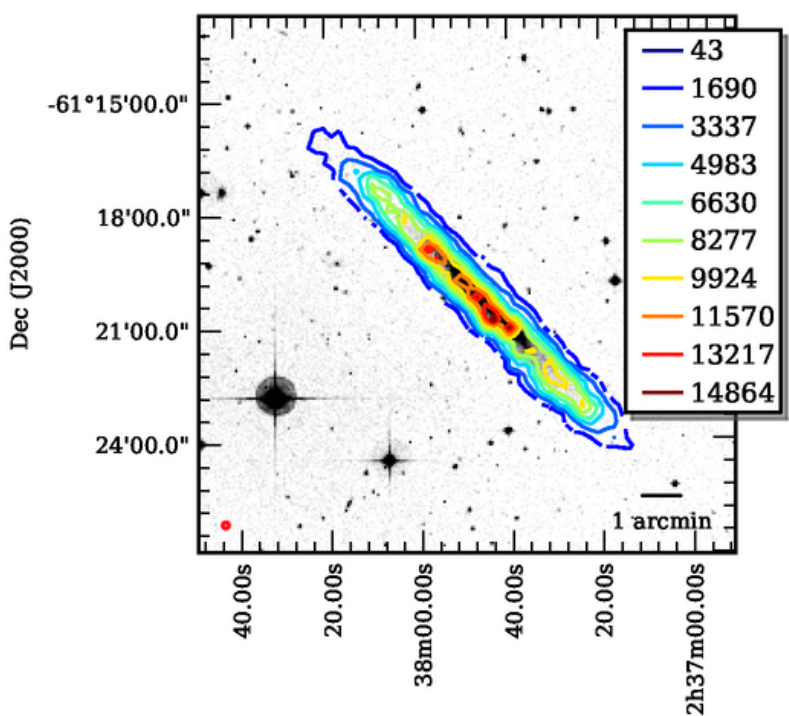

RA (J2000)

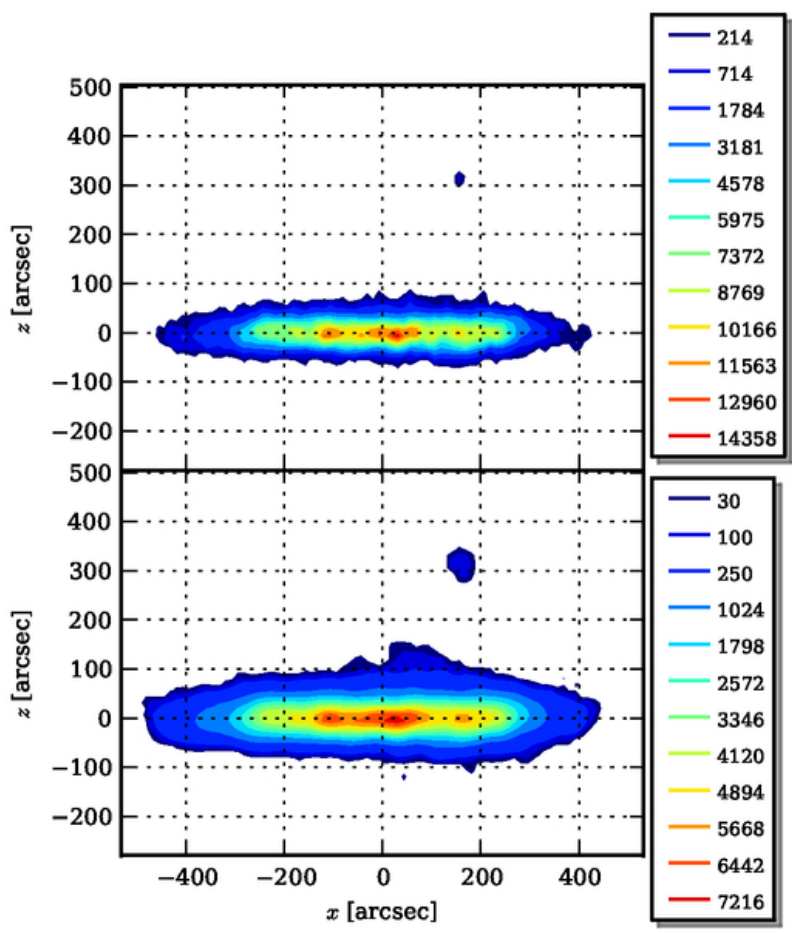

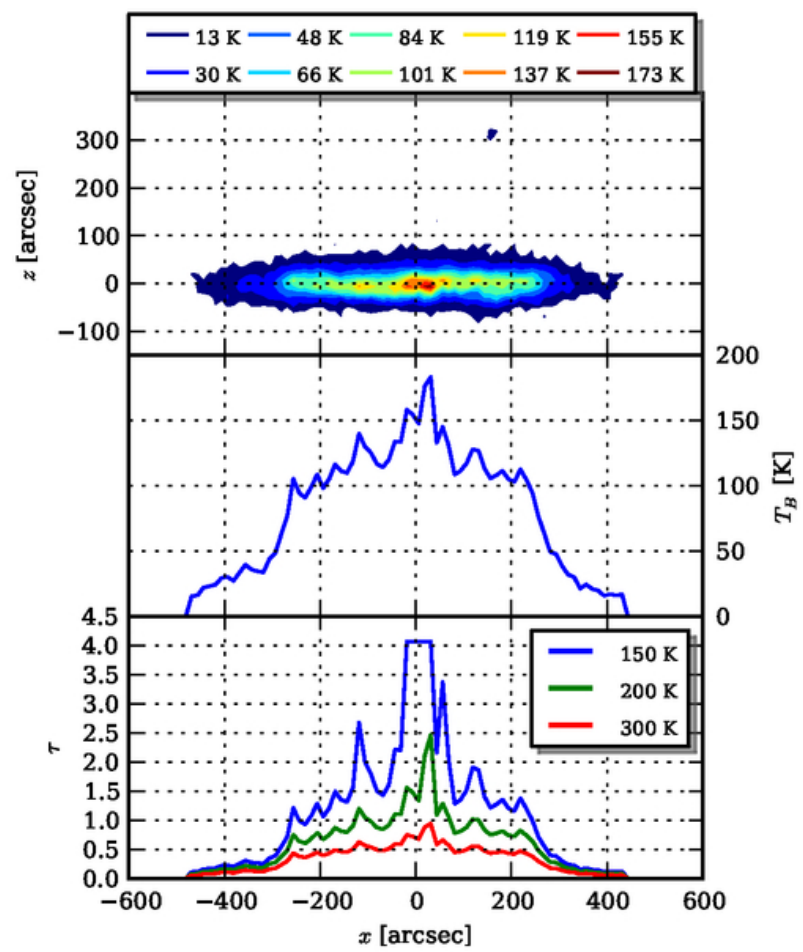

[Jy]

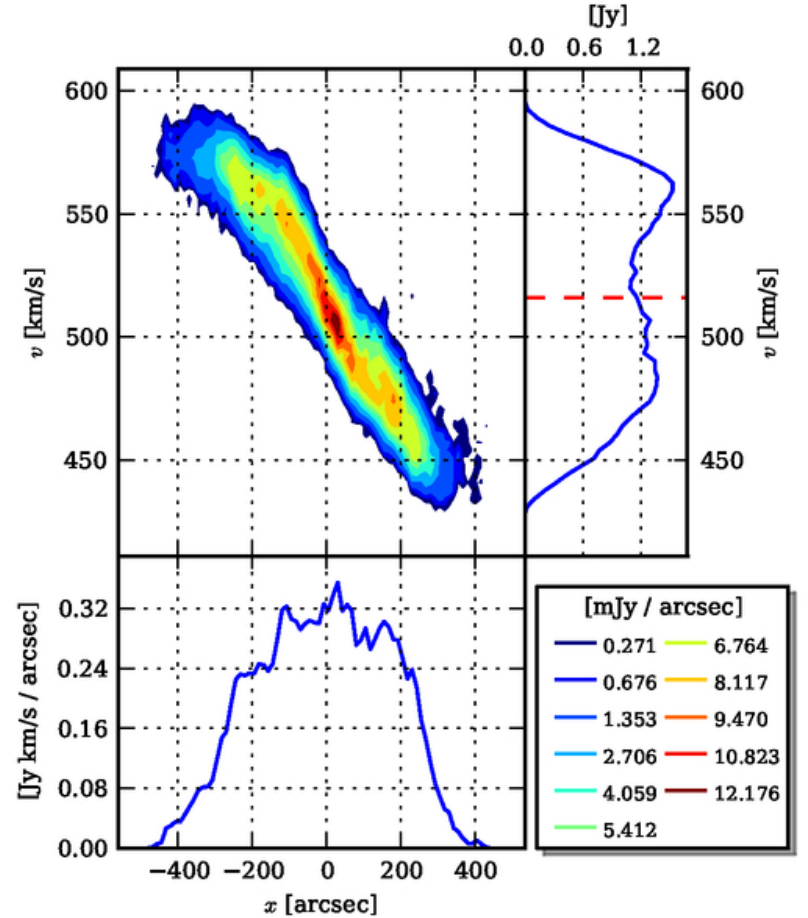

Figure 6. Overview of the neutral hydrogen contents of ESO 115-G021. Top-left: DSS1 overlaid with contours from the HI moment 0 map in $[\mathrm{K} /(\mathrm{km} / \mathrm{s})]$. Top-right: Top panel shows the maximum temperature along $v$. The middle panel shows the maximum temperature along $z$ with the same scale. The lower plot maximum temperature along $v$ and $z$ and shows the inferred self-absorption for that position, assuming a spin temperature of $125 \mathrm{~K}$. Bottom-left: Top panel shows the moment 0 for the high-resolution cube in [K/(km/s)]. Lower plot shows the moment 0 for the low-resolution cube. Bottom-left: Left panel shows the PV-diagram. Right-top panel shows the integrated flux per velocity. The red dashed line shows vsys. The lower plot shows the integrated flux per position along the major axis. 


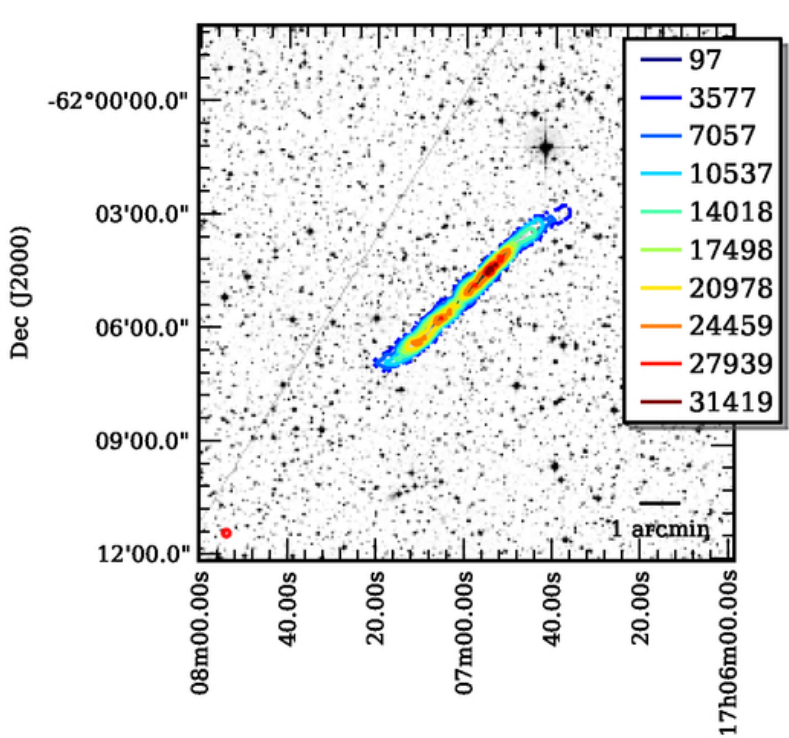

RA (J2000)

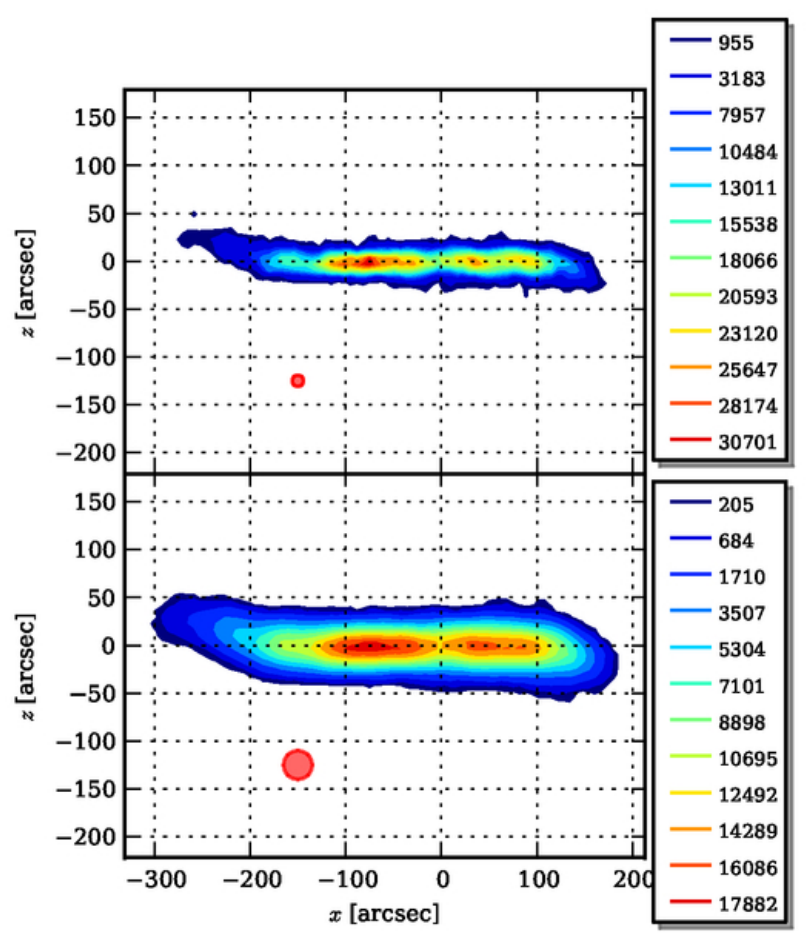

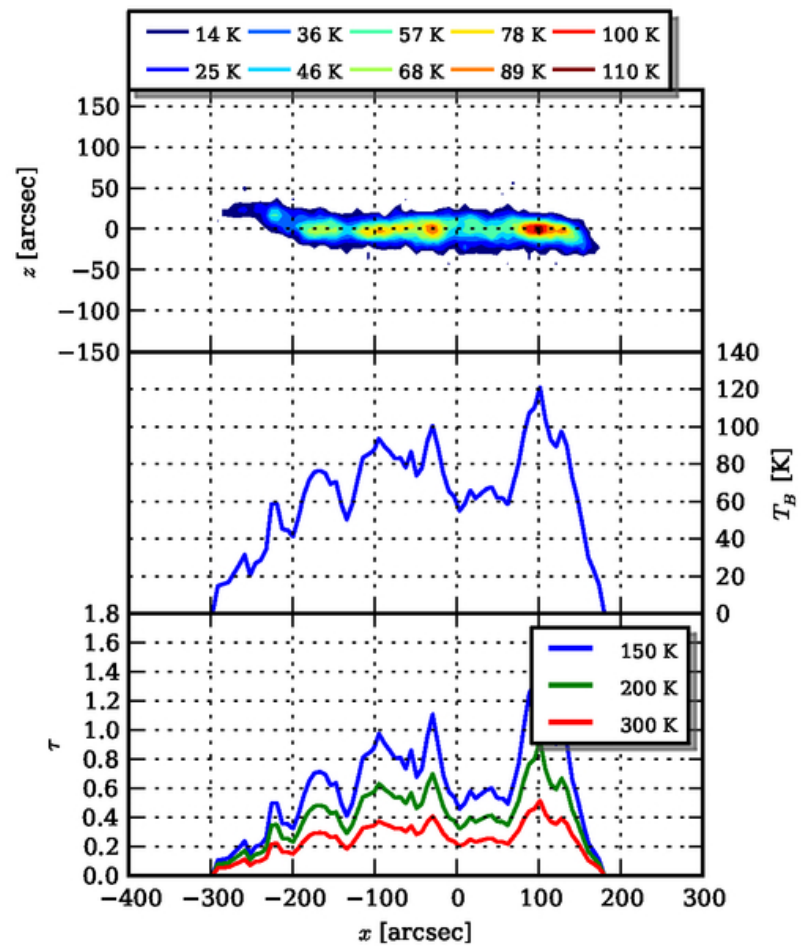

[Jy]

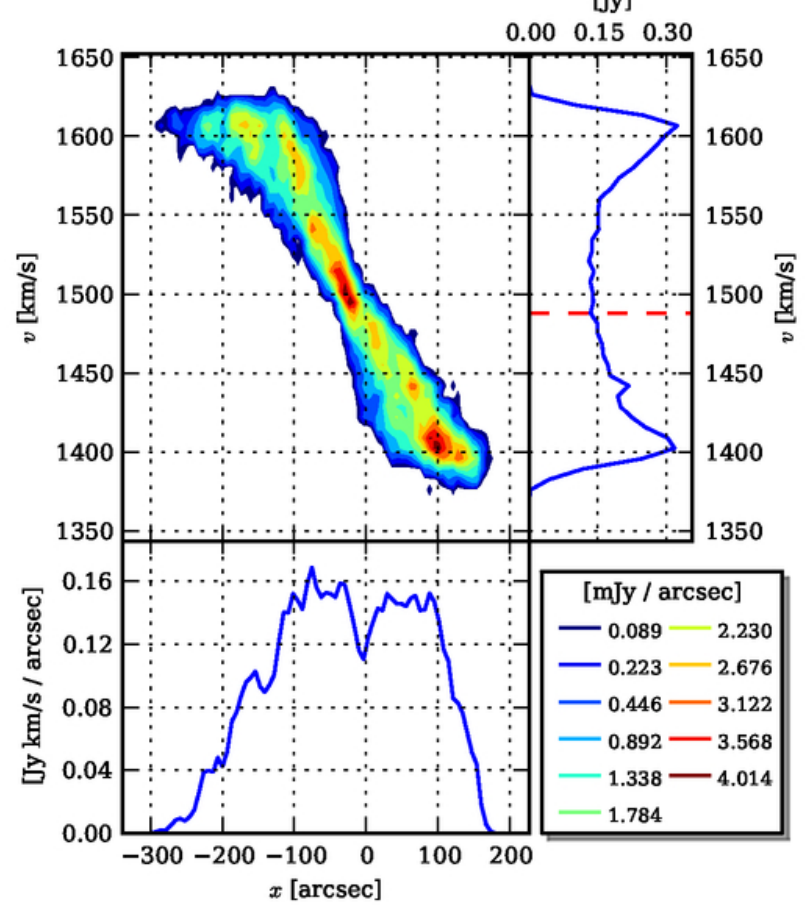

Figure 7. Overview of the neutral hydrogen contents of ESO 138-G014. Top-left: DSS1 overlaid with contours from the HI moment 0 map in $[\mathrm{K} /(\mathrm{km} / \mathrm{s})]$. Top-right: Top panel shows the maximum temperature along $v$. The middle panel shows the maximum temperature along $z$ with the same scale. The lower plot maximum temperature along $v$ and $z$ and shows the inferred self-absorption for that position, assuming a spin temperature of $125 \mathrm{~K}$. Bottom-left: Top panel shows the moment 0 for the high-resolution cube in [K/(km/s)]. Lower plot shows the moment 0 for the low-resolution cube. Bottom-left: Left panel shows the PV-diagram. Right-top panel shows the integrated flux per velocity. The red dashed line shows vsys. The lower plot shows the integrated flux per position along the major axis. 


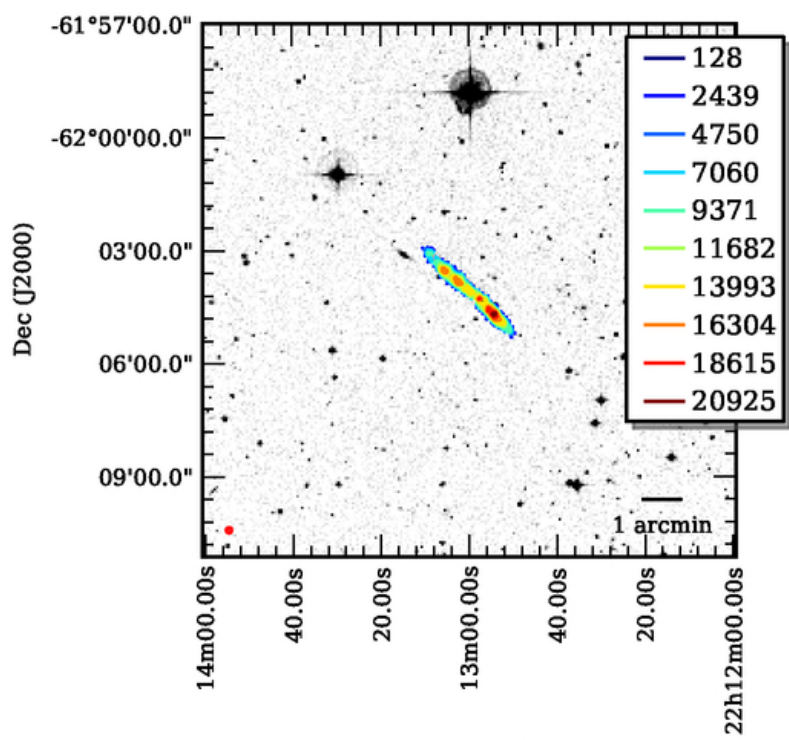

RA (J2000)

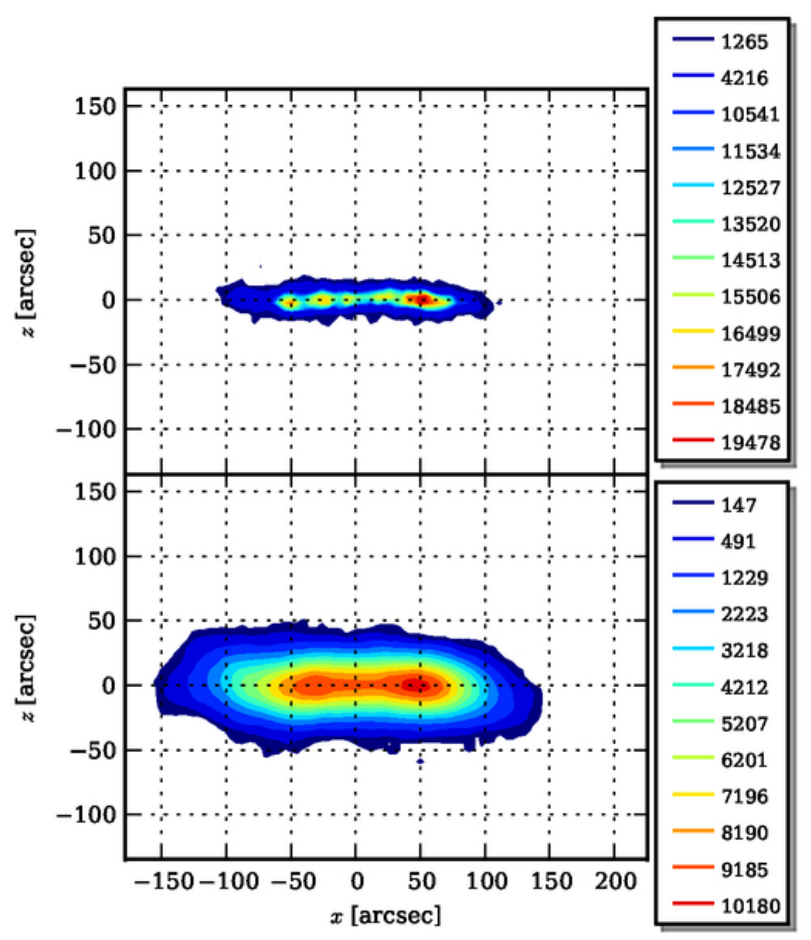

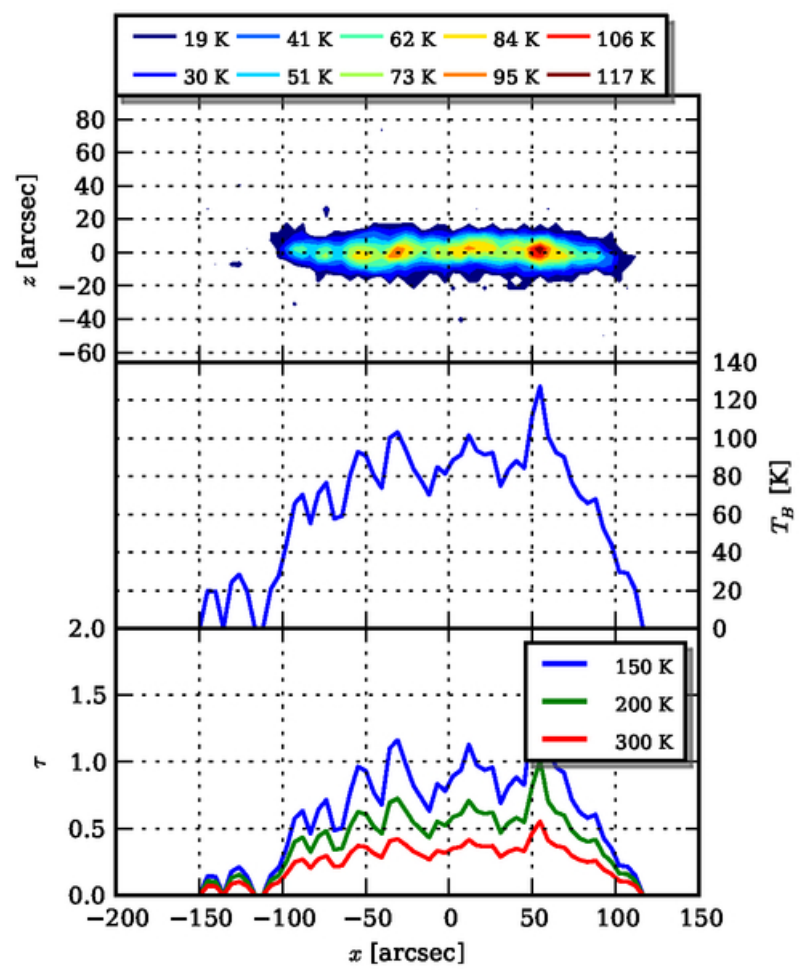

[Jy]

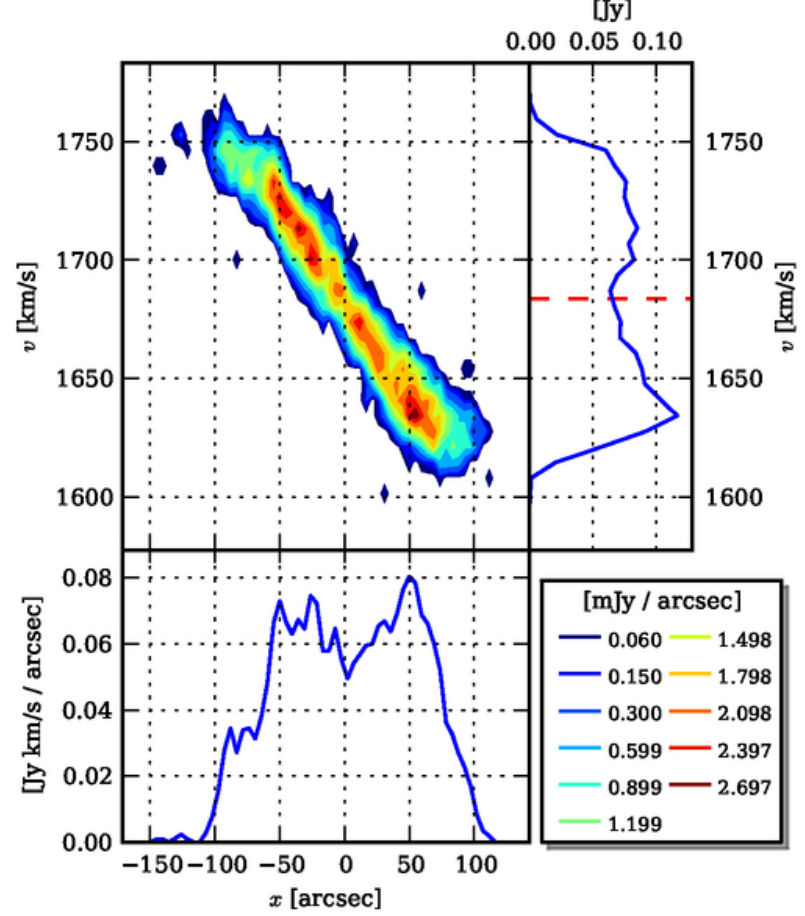

Figure 8. Overview of the neutral hydrogen contents of ESO 146-G014. Top-left: DSS1 overlaid with contours from the HI moment 0 map in $[\mathrm{K} /(\mathrm{km} / \mathrm{s})]$. Top-right: Top panel shows the maximum temperature along $v$. The middle panel shows the maximum temperature along $z$ with the same scale. The lower plot maximum temperature along $v$ and $z$ and shows the inferred self-absorption for that position, assuming a spin temperature of $125 \mathrm{~K}$. Bottom-left: Top panel shows the moment 0 for the high-resolution cube in [K/(km/s)]. Lower plot shows the moment 0 for the low-resolution cube. Bottom-left: Left panel shows the PV-diagram. Right-top panel shows the integrated flux per velocity. The red dashed line shows vsys. The lower plot shows the integrated flux per position along the major axis. 


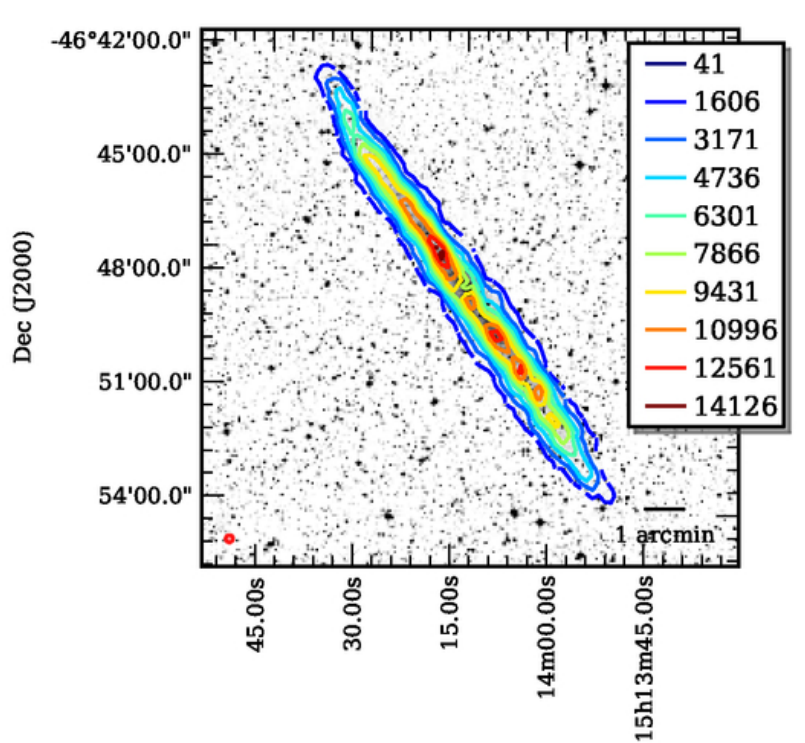

RA (J2000)

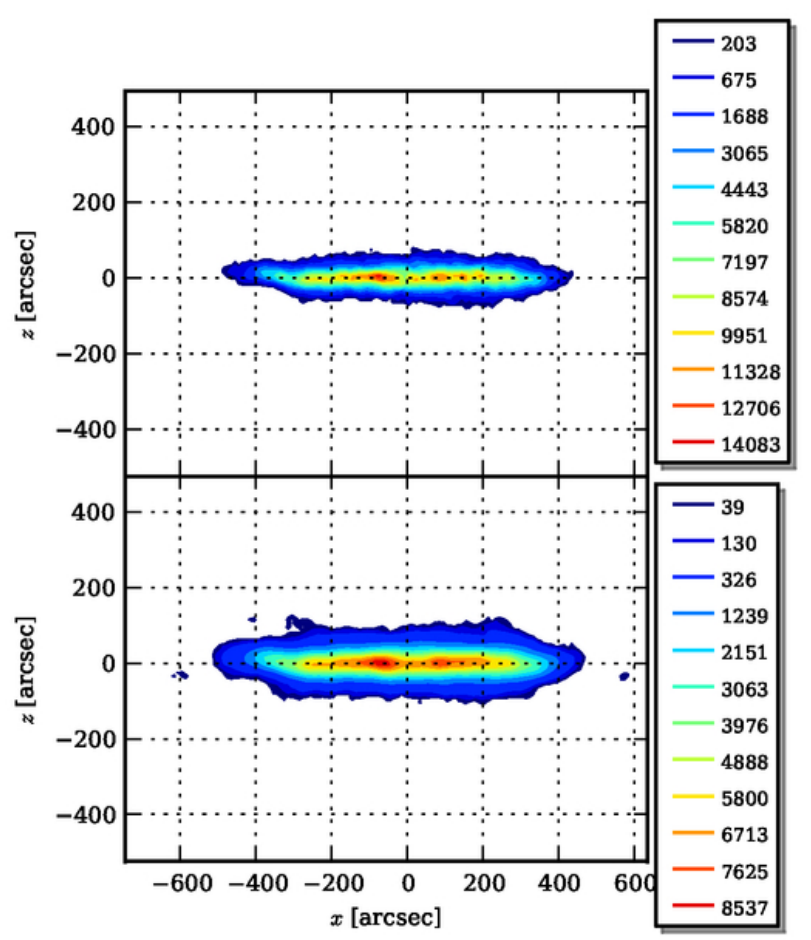

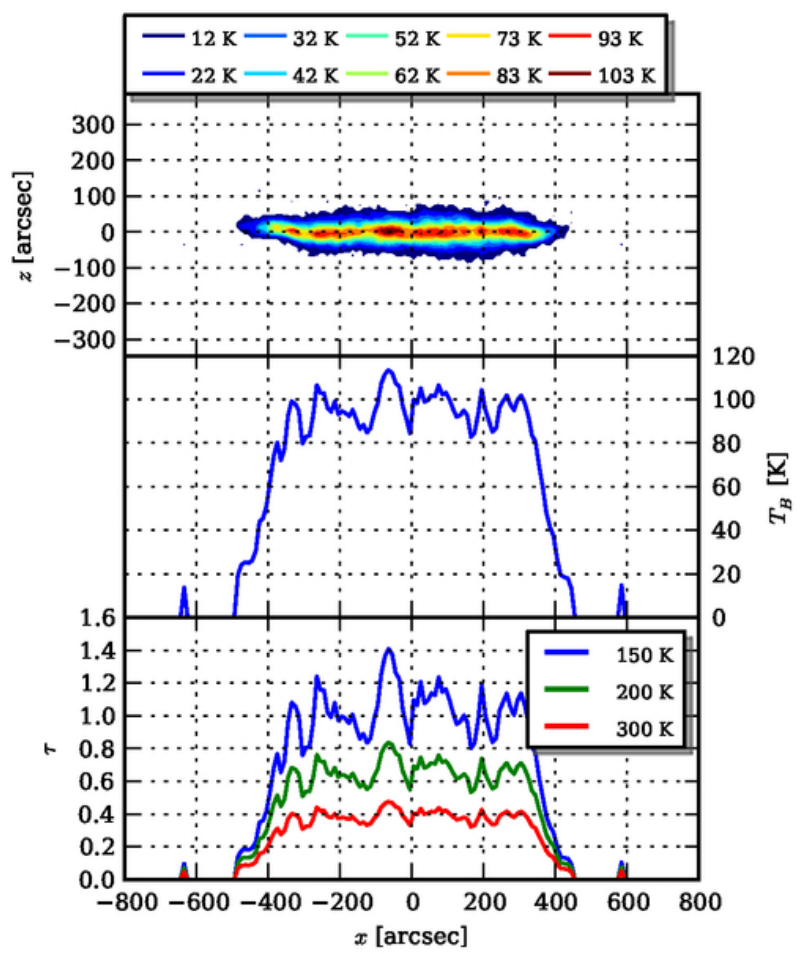

[Jy]

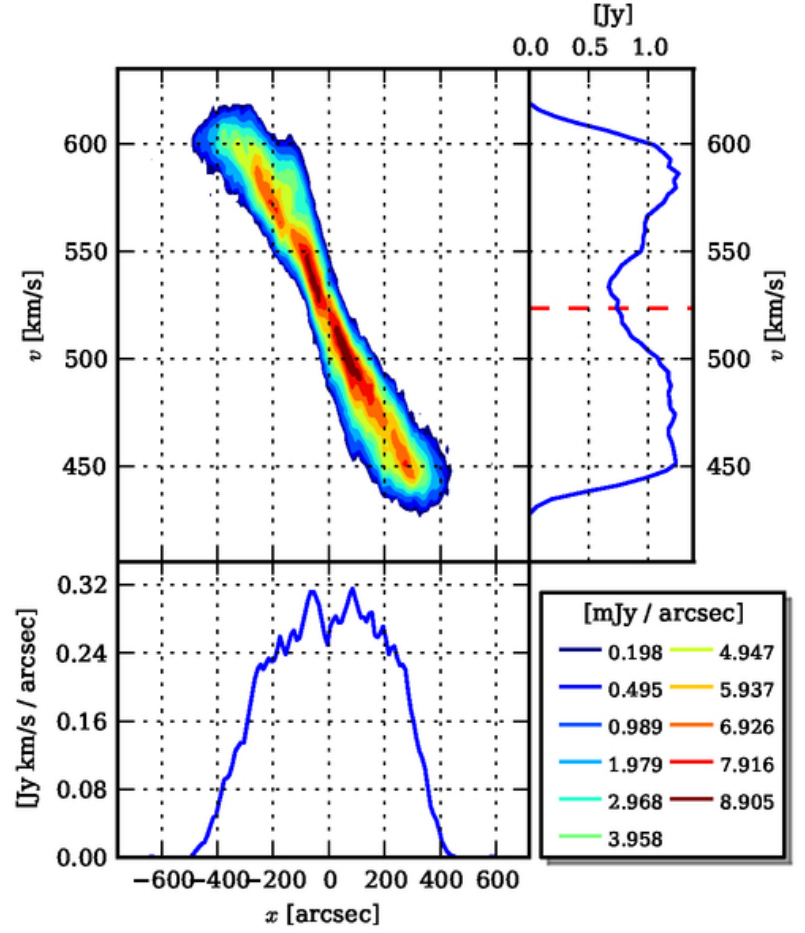

Figure 9. Overview of the neutral hydrogen contents of ESO 274-G001. Top-left: DSS1 overlaid with contours from the HI moment 0 map in $[\mathrm{K} /(\mathrm{km} / \mathrm{s})]$. Top-right: Top panel shows the maximum temperature along $v$. The middle panel shows the maximum temperature along $z$ with the same scale. The lower plot maximum temperature along $v$ and $z$ and shows the inferred self-absorption for that position, assuming a spin temperature of $125 \mathrm{~K}$. Bottom-left: Top panel shows the moment 0 for the high-resolution cube in [K/(km/s)]. Lower plot shows the moment 0 for the low-resolution cube. Bottom-left: Left panel shows the PV-diagram. Right-top panel shows the integrated flux per velocity. The red dashed line shows vsys. The lower plot shows the integrated flux per position along the major axis. 

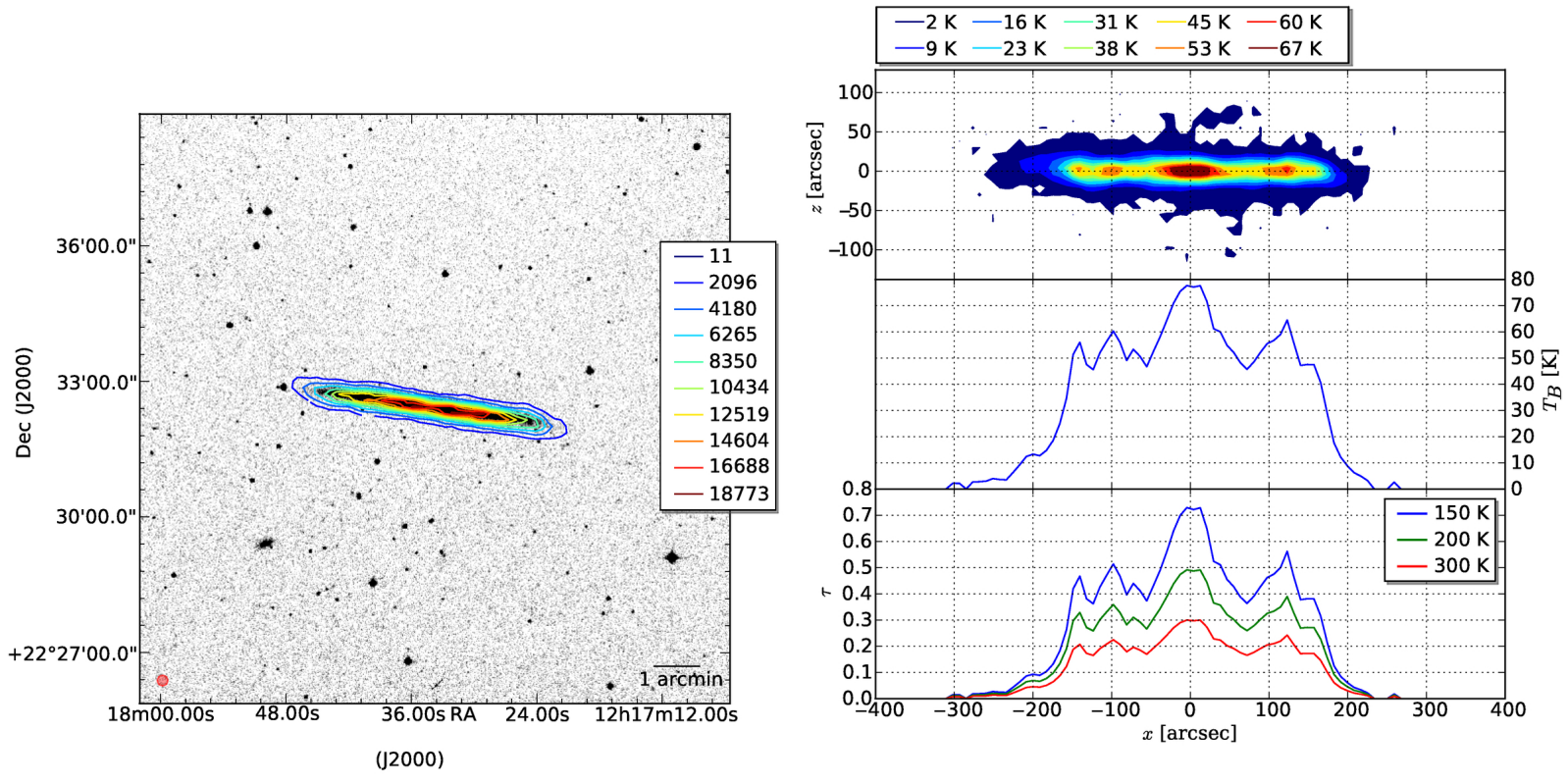

[Jy]
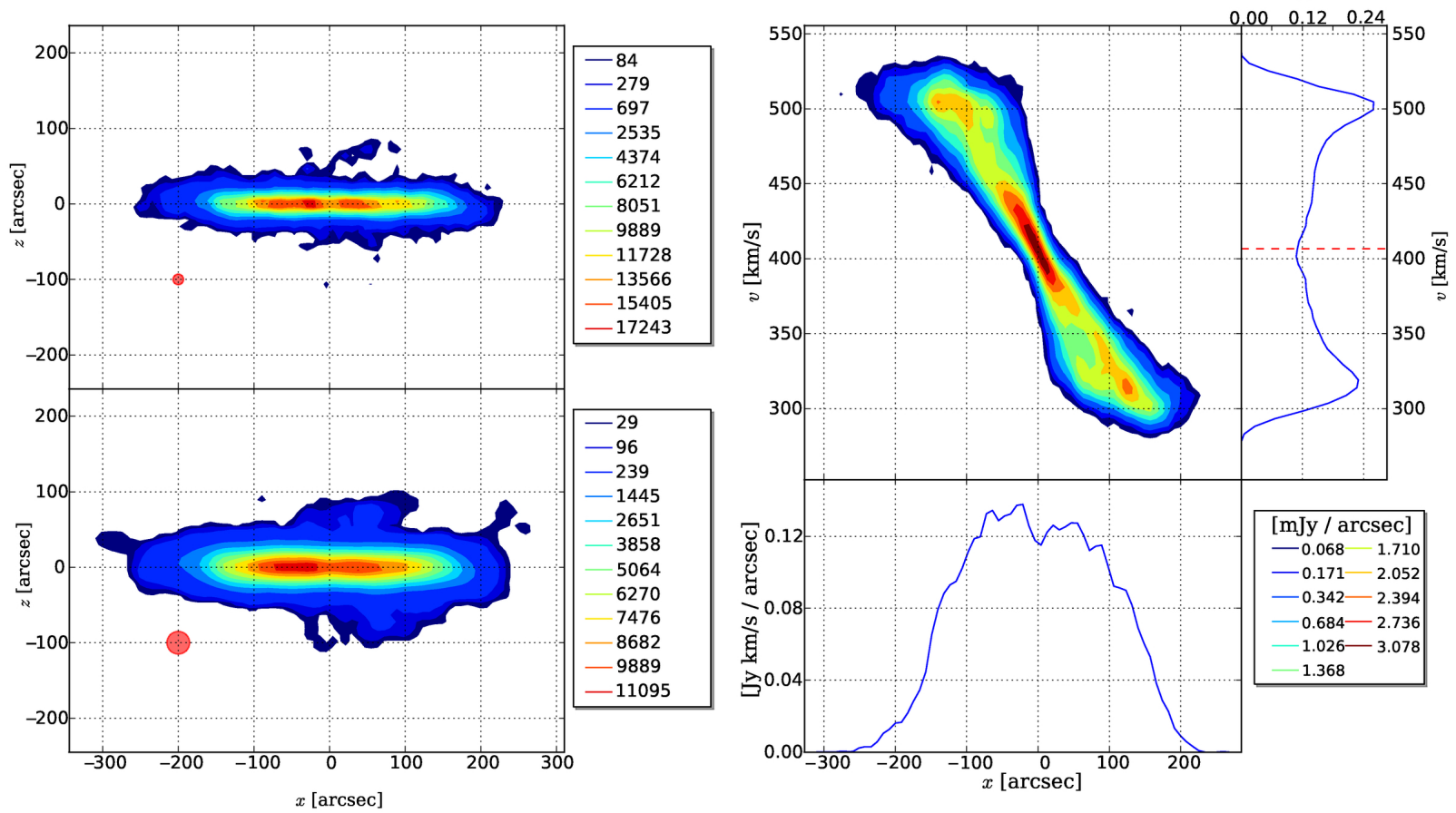

Figure 10. Overview of the neutral hydrogen contents of UGC 7321. Top-left: DSS1 overlaid with contours from the HI moment 0 map in $[\mathrm{K} /(\mathrm{km} / \mathrm{s})]$. Top-right: Top panel shows the maximum temperature along $v$. The middle panel shows the maximum temperature along $z$ with the same scale. The lower plot maximum temperature along $v$ and $z$ and shows the inferred self-absorption for that position, assuming a spin temperature of $125 \mathrm{~K}$. Bottom-left: Top panel shows the moment 0 for the high-resolution cube in $[\mathrm{K} /(\mathrm{km} / \mathrm{s})]$. Lower plot shows the moment 0 for the low-resolution cube. Bottom-left: Left panel shows the PV-diagram. Right-top panel shows the integrated flux per velocity. The red dashed line shows vsys. The lower plot shows the integrated flux per position along the major axis.

and S0 galaxies and star powered radio emission to starforming galaxies. AGN activity in low-luminosity galaxies seems low, although examples exist such as NGC 4395 (see the NASA/IPAC Extragalactic Database NED). It is likely

\footnotetext{
${ }^{4}$ We thank the referee to pointing out this system.
}

at the galactic centre (see Table 2 and we have therefore manually centred the galaxy on this position. Any other reasonable choice of the centre will require only a small shift, so that our results do not depend critically on this assumption.

AGN are known to exert strong influence on the ISM of the galaxy (e.g. Shulevski et al. (2012) and references 
therein). It is therefore interesting to note how regular the HI disc is, both in the zeroth-moment as in maximumtemperature maps. Most of the inner disc has a maximum surface brightness temperature that forms a remarkably flat plateau between 80 and $100 \mathrm{~K}$ (Figure 9 bottom-right). The galaxy is barely visible in most optical and infrared bands, indicating the presence of only little stellar mass. This may explain why the gas is relatively undisturbed, in comparison with our other galaxies. The galaxy is known to have modest star formation at $1.5 \times 10^{-2} \mathrm{M}_{\odot}$ per year (Côté et al. 2009).

There is a warp visible beyond $\sim 280$ arcsec on both sides of the galaxy (Figure 9 bottom-left). The XV-diagram is slightly asymmetric, with more gas at the low velocity side. This is also visible in the integrated profiles (Figure 9 bottom-right). The shape of the spectrum matches well to HIPASS, although it is more symmetric.

\subsection{UGC 7321}

UGC 7321 is a well-known low surface brightness galaxy, which has already been studied using the Westerbork Synthesis Radio Telescope (WSRT) by García-Ruiz et al. (2002) and the VLA by Uson \& Matthews (2003). The observations by Uson \& Matthews (2003) form the basis of our work here. In terms of noise levels, it is the best-resolved galaxy in our sample. It is also our only northern-sky galaxy.

The zeroth-moment map in Figure 10 bottom-left shows a well-behaved, symmetric galaxy. Only at large radii is there some indication of warping, a feature already noted by Matthews \& Wood (2003), who also notes it is flaring. Additionally, they find evidence that suggests the presence of an HI halo. We believe that this halo might be visible as the extended structure on the lower figure, between 0 and 110 arcseconds.

The XV-diagram of the galaxy is asymmetric. The receding side clearly exhibits a flat rotation curve, but in contrast the approaching side continues to rise over much of the observed extent of the galaxy.

The maximum surface brightness map shows a clear central peak and two additional peaks around 100 arcseconds (Figure 10 bottom-right). These peaks appear to be symmetric around the centre of the galaxy. The rest of the galaxy forms a plateau, around $60 \mathrm{~K}$. We again see that the maximum temperature trails slowly off into the warp on the right-hand side.

\section{DISCUSSION}

The beam of the high resolution cubes is on average 1-2" larger than O'Brien et al. (2010) (Table 3). This is due to most of the new observations being taken in tight baseline configurations. There are therefore more short baselines. The weight in the UV-plane shifts to slightly smaller scales, resulting in larger beams. The effect is small enough to be of little importance to this work. In the high resolution cubes, the noise is significantly lower, thanks to the additional observations and larger beams. Where no additional observations were available, the noise is (as expected) roughly equal.

Overall, there is good agreement with O'Brien et al. (2010) on the derived central positions, even though we include more faint extended emission in our measurements.
The biggest offset is in ESO 138-G014 (see Figure 7 bottomleft), where the non-symmetric warp is affecting the position estimate. The position angles however do agree well, except that in some cases we have added an additional $180^{\circ}$ because we align the galaxies such that the approaching side is aligned to the right-hand side. Similarly, the systemic velocities vsys and associated parameters $W 20, W 50$ and vmax are all well determined. Kinematic modelling will have to be performed to find the true centre of each galaxy, the derived values here will only serve as first estimates for the subsequent analysis in Paper III.

We have also compared the integrated flux $F I$ with the HIPASS catalogue (Doyle et al. 2005) and the work by O'Brien et al. (2010). In general our results match well to HIPASS. ESO 274-G001 has the largest difference, with 25 mJy $\mathrm{km} / \mathrm{s}$ more detected here than in HIPASS. The other major differences are for ESO 115-G021 with a difference of $19 \mathrm{mJy} \mathrm{km} / \mathrm{s}$, and IC 5052 with a difference of $7 \mathrm{mJy} \mathrm{km} / \mathrm{s}$. In both cases our observed flux again is higher.

These three galaxies are the largest in the sample. The Parkes single dish beam has a angular resolution of 15.5 arcminutes. Most likely, the Parkes beam fails to cover the entire galaxy, missing the outskirts and is therefore getting a lower total flux. With that correction in mind, each data cube has successfully recovered all flux from HIPASS.

O'Brien et al. (2010) report very different values for $F I$. Most striking is IC 5052, where only $37.9 \mathrm{mJy} \mathrm{km} / \mathrm{s}$ is reported, against $117.7 \mathrm{mJy} \mathrm{km} / \mathrm{s}$ here. It is unclear to us why O'Brien et al. (2010) often reports very different values. Most likely, it has to do with the masking of extended emission in their work. Indeed, our value agrees well with the $\sim 100 \mathrm{mJy} \mathrm{km} / \mathrm{s}$ derived by Hong et al. (2013).

As noted before, the distances to the galaxies are assumed further than in O'Brien et al. (2010). This revision is mostly due to new observations, which use the tip of the red giant branch or the Tully-Fisher relation. This allows us to use cosmology independent distances for all galaxies. That change, plus the higher values for $F I$, combine to have a large impact on the inferred total mass $M_{\mathrm{HI}}$ of the galaxy. Most galaxies now have estimated HI masses anywhere between 20 and $300 \%$ higher.

All eight of our galaxies shows signs of warping. This is consistent with the work by García-Ruiz et al. (2002), who found that all galaxies with extended HI disc have warps. All of the warps occur at or just beyond the visible stellar disc, similar to the findings by van der Kruit (2007). The only possible exception to this rule could be ESO 146-G14, which the warp in the HI sets in on the sky well before the end of the stellar disk (see Figure 8 top-left). This is most likely due to the largest amplitude of the warp occuring nearer to the line of sight.

\subsection{Self-absorption}

As we pointed out in Section 3 the $21-\mathrm{cm}$ line of $\mathrm{HI}$ is known to be optically thick along many sight lines in our own (edge-on) Galaxy (Allen et al. 2012), and similar results have been found in M 31 and M 33 (Braun 2012). Such self-absorption of the HI emission not only biases the total hydrogen masses, it also raises questions about the use of the HI velocity structure for studies of the gas kinematics. Nowhere are the answers to such questions more urgent than 
The Shape of Dark Matter Haloes I.

in the study of highly inclined galaxies, where techniques have been proposed to "peel off" the edges of the observed $\mathrm{HI}$ in order to expose the detailed velocity dispersion of the ISM over large segments of the galaxy discs in both $R$ and $z$ (O'Brien et al. 2010a).

If a moderately inclined galaxy as M 31 hides $34 \%$ of its HI mass (Braun 2012), what is the effect on edge-on galaxies? In the neutral hydrogen, the dominating factor for selfabsorption would be the spin temperature of the cold neutral medium, which would serve as an upper limit to the observable surface brightness temperature. Consider now the maximum surface brightness maps for ESO 274-G001 (Figure 9 bottom-right). There is a clear plateau around 90 Kelvin throughout almost the entire galaxy. At the outskirts the line of sight are much shorter than near the inner parts, yet the observed peak temperature remains stable. A similar behavior can be seen in all other galaxies in our sample. As a case of even shorter path-lengths, consider Allen et al. (2012, Figure 3), where a 'mini-survey' has been performed of $\mathrm{HI}, \mathrm{OH}$ and $\mathrm{CO}$ at 30' resolution inside the Galaxy. The observed gas is local, within about $2 \mathrm{kpc}$ of the sun. The peak temperature of the $\mathrm{HI}$ is again $90 \mathrm{~K}$, only this time we are seeing the individual clouds. Why would such different path-lengths always result in the same brightness temperature?

The traditional view is that at these brightness temperatures, column densities are reached at which the atomic gas starts to be converted into molecular gas, thus providing a natural threshold to the observed temperatures (Stecher \& Williams 1967. Hollenbach et al. 1971. Federman et al. 1979). However, a column density is an artificial construct based on the position of the observer, and as such, the gas cannot be expected to conform to it. A volume density threshold would seem to be a much more physically relevant quantity. However, since the path lengths between the local HI clouds from Allen et al. (2012) and the central parts of the edge-on galaxies presented here vary over roughly two orders of magnitude, the volume and the inferred density are also likely to vary in the same way. Any volume density threshold would seem to have difficulty explaining the close similarities in the maximum brightness temperatures.

Opacity however can explain this naturally, as we demonstrated in Section 3 The brightness temperature of 90 Kelvin is in the same range as the median spin temperature (Dickey \& Lockman 1990 Dickey et al. 2009, Draine 2011). $90 \mathrm{~K}$ is then the maximum attainable temperature before the gas turns optically thick. We are thus seeing optically thick gas in Allen et al. (2012). In Figure 9 bottom-right, the path-lengths have become so long compared to the sizes of the clouds, that each line is bound to hit an optically thick cloud. Considering the radii at which these can be seen, a lot more than $30 \%$ of the galaxy HI mass could be hidden in and behind these clouds.

The self-absorption will also influence the observed kinematics and structure of the disc. As the brightest parts are of the gas are scaled down by the self-absorption, a fit to the thickness of the disc can be deceived into detecting a thicker disc than is actually present. Similarly the velocity dispersion can appear larger than it would have been had the gas been optically thin. Because of these issues, we do not model the galaxies in this paper, but will first develop software that can model self-absorption and that will be the subject of Paper II.

\section{ACKNOWLEDGMENTS}

SPCP is grateful to the Space Telescope Science Institute, Baltimore, USA, the Research School for Astronomy and Astrophysics, Australian National University, Canberra, Australia, and the Instituto de Astrofisica de Canarias, La Laguna, Tenerife, Spain, for hospitality and support during short and extended working visits in the course of his $\mathrm{PhD}$ thesis research. He thanks Roelof de Jong and Ron Allen for help and support during an earlier period as visiting student at Johns Hopkins University and the Physics and Astronomy Department, Krieger School of Arts and Sciences for this appointment.

PCK thanks the directors of these same institutions and his local hosts Ron Allen, Ken Freeman and Johan Knapen for hospitality and support during many work visits over the years, of which most were directly or indirectly related to the research presented in this series op papers.

Work visits by SPCP and PCK have been supported by an annual grant from the Faculty of Mathematics and Natural Sciences of the University of Groningen to PCK accompanying of his distinguished Jacobus C. Kapteyn professorhip and by the Leids Kerkhoven-Bosscha Fonds. PCK's work visits were also supported by an annual grant from the Area of Exact Sciences of the Netherlands Organisation for Scientific Research (NWO) in compensation for his membership of its Board.

\section{REFERENCES}

Allen R. J., Ivette Rodríguez M., Black J. H., et al., 2012, AJ, 143, 97

Ann H. B., Park J.-C., 2006, NewAstr., 11, 293

Athanassoula E., 2016, In; Galactic Bulges, E. Laurikainen, R. Peletier, D. Gadotti (Eds.), ASSL 418, p.391. Springer

Bland-Hawthorn J., 2014, The Origin of the Galaxy and Local Group, Appendix A, p.109. Oxford Univ. Press.

Braun R., 2012, ApJ, 749, 87

Bureau M., Aronica G., Athanassoula E., et al. 2006, MNRAS, 370, 753

Bureau M., Freeman K. C., 1997, Pub. Astr. Soc. Austral., 14, 146

Bureau M., Freeman K. C., 1999, AJ, 118, 126

Burstein D., 1979, ApJ, 234, 829

Comerón S., Knapen J. H., Sheth K., et al., 2011, ApJ, 729, 18

Condon J. J., Cotton W. D., Broderick J. J., 2002, AJ, 124, 675

Côté S., Draginda A., Skillman E. D., Miller B. W., 2009, AJ, 138, 1037

de Geyter G., Baes M., Camps P., et al., 2013, A\&A, 550, A74

de Vaucouleurs G., de Vaucouleurs A., Corwin Jr. H. G., et al., 1991, Third Reference Catalogue of Bright Galaxies. Springer-Verlag, Berlin.

Dickey J. M., Lockman F. J., 1990, ARA\&A, 28, 215 
Dickey J. M., Strasser S., Gaensler B. M., et al., 2009, ApJ, 693,1250

Douglas K. A., Acreman D. M., Dobbs C. L., et al., 2010, MNRAS, 407, 405

Doyle M. T., Drinkwater M. J., Rohde D. J., et al' 2005, MNRAS, 361, 34

Draine B. T., 2011, Physics of the Interstellar and Intergalactic Medium. Princeton Univ. Press.

Federman S. R., Glassgold A. E., Kwan J., 1979, ApJ, 227, 466

Freeman K. C., 1970, ApJ, 160, 811

García-Ruiz I., Sancisi R., Kuijken K., 2002, A\&A, 394, 769

Gentile G., Fraternali F., Klein U., Salucci P., 2003, A\&A, 405, 969

Gibson S. J., Taylor A. R., Higgs L. A., et al., 2005, ApJ, 626, 195

Gilmore G., Reid N., 1983, MNRAS, 202, 1025

Hollenbach D. J., Werner M. W., Salpeter E. E., 1971, ApJ, 163, 165

Hong T., Staveley-Smith L., Masters K. L., et al. 2013, MNRAS, 432, 1178

Jarrett T. H., Chester T., Cutri R., et al., 2003, AJ, 125, 525

Koribalski B. S., 2008, The Local Volume HI Survey (LVHIS). p. 41

Lewis B. M., 1984, ApJ, 285, 453

Matthews L. D., 2000, The Astronomical Journal, 120, 1764

Matthews L. D., Wood K., 2003, ApJ, 593, 721

Mihalas D., Binney J., 1981, Galactic astronomy: Structure and kinematics /2nd edition/. Freeman \& Co., San Francisco

O’Brien J. C., Freeman K. C., van der Kruit P. C., 2010a, A\&A, 515, A61

O'Brien J. C., Freeman K. C., van der Kruit P. C., 2010b, A\&A, 515, A62

O'Brien J. C., Freeman K. C., van der Kruit P. C., 2010c, A\&A, 515, A63

O'Brien J. C., Freeman K. C., van der Kruit P. C., Bosma A., 2010, A\&A, 515, A60

Olling R. P., 1996, AJ, 112, 457

Oosterloo T., Fraternali F., Sancisi R., 2007, AJ, 134, 1019

Peters S. P. C., 2014, PhD thesis, Univ. Groningen

Peters S. P. C., van der Kruit P. C., Knapen J. H., et al., 2015, MNRAS, submitted, .

Pohlen M., Zaroubi S., Peletier R., Dettmar R.-J., 2007, Mon.Not.Roy.Astron.Soc., 378, 594

Radburn-Smith D. J., de Jong R. S., Seth A. C., et al., 2011, ApJS, 195, 18

Rupen M. P., 1991, AJ, 102, 48

Saha K., Levine E. S., Jog C. J., et al., 2009, ApJ, 697, 2015

Sánchez-Saavedra M. L., Battaner E., Guijarro A., et al., 2003, A\&A, 399, 457

Sancisi R., 1976, A\&A, 53, 159

Sancisi R., Allen R. J., 1979, A\&A, 74, 73

Sault R. J., Teuben P. J., Wright M. C. H., 1995, in Astronomical Data Analysis Software and Systems IV, Vol. 77 of Astron. Soc. Pac. Conf. Ser., . p. 433

Shulevski A., Morganti R., Oosterloo T., Struve C., 2012, A\&A, 545, A91
Skrutskie M. F., Cutri R. M., Stiening R., et al., 2003, VizieR Online Data Catalog, 7233, 0

Stecher T. P., Williams D. A., 1967, ApJl, 149, L29

Swaters R. A., Sancisi R., van der Hulst J. M., 1997, ApJ, 491, 140

Taylor A. R., Jansen R. A., Windhorst R. A., et al., 2003, AJ, 125, 3145

Tsikoudi V., 1980, ApJS, 43, 365

Tully R. B., Shaya E. J., Karachentsev I. D., et al., 2008, ApJ, 676, 184

Uson J. M., Matthews L. D., 2003, AJ, 125, 2455

van der Kruit P. C., 1979, A\&AS, 38, 15

van der Kruit P. C., 1981, A\&A, 99, 298

van der Kruit P. C., 1984, A\&A, 140, 470

van der Kruit P. C., 2007, A\&A, 466, 883

van der Kruit P. C., Freeman K. C., 2011, ARA\&A, 49, 301

van der Kruit P. C., Jiménez-Vicente J., Kregel M., Freeman K. C., 2001, A\&A, 379, 374

van der Kruit P. C., Searle L., 1981, A\&A, 95, 105

Warmels R. H., 1988, A\&AS, 72, 427

Weliachew L., Sancisi R., Guelin M., 1978, A\&A, 65, 37

Wright A. E., Griffith M. R., Burke B. F., Ekers R. D., 1994, ApJ Suppl., 91, 111

This paper has been typeset from a $\mathrm{T}_{\mathrm{E}} \mathrm{X} / \mathrm{LAT}_{\mathrm{E}} \mathrm{X}$ file prepared by the author. 